\title{
Outgassing on stagnant-lid super-Earths
}

\author{
C. Dorn ${ }^{1}$, L. Noack ${ }^{2,3}$, and A. B. Rozel ${ }^{4}$ \\ ${ }^{1}$ Institute of Computational Sciences, University of Zurich, Winterthurerstrasse 109, 8057, Zurich, Switzerland \\ e-mail: cdorn@physik.uzh.ch \\ ${ }^{2}$ Department of Reference Systems and Geodynamics, Royal Observatory of Belgium, Avenue Circulaire 3, 1180 Brussels, \\ Belgium \\ ${ }^{3}$ Institute of Geological Sciences, Free University Berlin, Malteserstr. 74-100, 12249 Berlin, Germany \\ ${ }^{4}$ Institute of Geophysics, Department of Earth Sciences, ETH Zurich, Sonneggstrasse 5, 8092 Zurich, Switzerland
}

Received 5 July 2017 / Accepted 25 February 2018

\begin{abstract}
Aims. We explore volcanic $\mathrm{CO}_{2}$-outgassing on purely rocky, stagnant-lid exoplanets of different interior structures, compositions, thermal states, and age. We focus on planets in the mass range of 1-8 $M_{\oplus}$ (Earth masses). We derive scaling laws to quantify first- and second-order influences of these parameters on volcanic outgassing after $4.5 \mathrm{Gyr}$ of evolution.

Methods. Given commonly observed astrophysical data of super-Earths, we identify a range of possible interior structures and compositions by employing Bayesian inference modeling. The astrophysical data comprise mass, radius, and bulk compositional constraints; ratios of refractory element abundances are assumed to be similar to stellar ratios. The identified interiors are subsequently used as input for two-dimensional (2D) convection models to study partial melting, depletion, and outgassing rates of $\mathrm{CO}_{2}$.

Results. In total, we model depletion and outgassing for an extensive set of more than 2300 different super-Earth cases. We find that there is a mass range for which outgassing is most efficient $\left(\sim 2-3 M_{\oplus}\right.$, depending on thermal state) and an upper mass where outgassing becomes very inefficient ( $\sim 7-7 M_{\oplus}$, depending on thermal state). At small masses (below 2-3 $M_{\oplus}$ ) outgassing positively correlates with planet mass, since it is controlled by mantle volume. At higher masses (above 2-3 $M_{\oplus}$ ), outgassing decreases with planet mass, which is due to the increasing pressure gradient that limits melting to shallower depths. In summary, depletion and outgassing are mainly influenced by planet mass and thermal state. Interior structure and composition only moderately affect outgassing rates. The majority of outgassing occurs before $4.5 \mathrm{Gyr}$, especially for planets below $3 M_{\oplus}$.

Conclusions. We conclude that for stagnant-lid planets, (1) compositional and structural properties have secondary influence on outgassing compared to planet mass and thermal state, and (2) confirm that there is a mass range for which outgassing is most efficient and an upper mass limit, above which no significant outgassing can occur. Our predicted trend of $\mathrm{CO}_{2}$-atmospheric masses can be observationally tested for exoplanets. These findings and our provided scaling laws are an important step in order to provide interpretative means for upcoming missions such as JWST and E-ELT, that aim at characterizing exoplanet atmospheres.
\end{abstract}

Key words. planets and satellites: tectonics - planets and satellites: terrestrial planets - planets and satellites: atmospheres - planets and satellites: interiors

\section{Introduction}

Super-Earths are among the most abundant exoplanets and are characterized by small volatile fractions (e.g., Dressing \& Charbonneau 2015; Fulton et al. 2017). SuperEarths have planet masses and radii that exceed the diversity of the Solar System planets (Fig. 1). Our knowledge of the variability of their interiors is limited because data (e.g., mass and radius) are few and allow for very different interior structures and compositions. The only parts of exoplanets that can be directly probed are their atmospheres. So far, there are only a few small-mass planets (GJ1214b, HD97658b, 55Cnc e, GJ1132b) for which constraints on their atmospheres are available. However, near future spectroscopic observations (e.g., E-ELT, JWST, ARIEL) will allow us to gain detailed insights into the atmospheric compositions for a number of super-Earths.

The anticipated diversity of atmospheres on super-Earth exoplanets is subject to planet formation and evolution processes (Leconte et al. 2015). Different processes can shape the thickness and chemical make-up of an atmosphere: gas accretion from the stellar nebular, atmospheric enrichment by the disruption of planetesimals, outgassing from an early magma ocean or long-term out- and in-gassing processes, and hydrodynamic escape. The understanding of these processes is crucial for the interpretation of atmospheric characteristics inferred from observations. Here, we focus on volcanic outgassing that can constantly release volatiles on geological timescales into the atmosphere that were once trapped in the mantle. Volcanic outgassing can be the origin of enriched atmospheres. Dorn \& Heng (2018) identified these to be likely dominating those planets of small masses and warm to hot equilibrium temperatures. The importance of volcanic outgassing on observed super-Earths is the subject of ongoing research. We anticipate that the diversity in planetary interiors and thermal states may significantly influence volcanic activity and consequently the thicknesses of outgassed atmospheres, which we address in this study.

The diversity in interior structures and compositions for observed exoplanets is generally expected to be large. For rocky exoplanets, despite the given data of planetary masses and radii, 


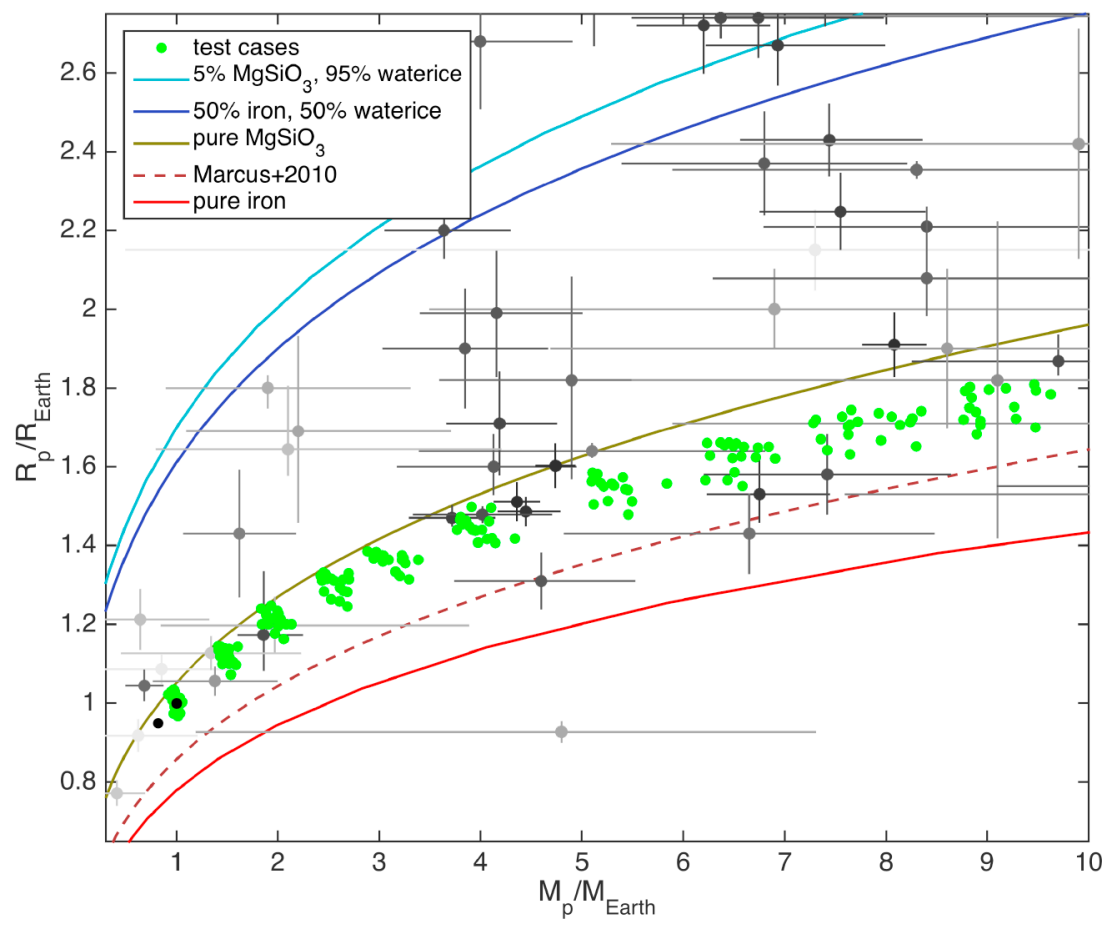

Fig. 1. Mass-radius diagram for planets below $2.7 R_{\oplus}$ and $10 M_{\oplus}$ (180 Super-Earths shown). Transparencies of the black points scale with the relative error on planet mass. Green dots represent synthetic planets used in our study. The dashed curve denotes the minimum radius predicted for maximum mantle stripping due to giant impacts (Marcus et al. 2010). there is significant ambiguity on possible core sizes and mantle compositions. This ambiguity can be significantly reduced by accounting for possible correlations between stellar and planetary compositions, specifically their relative abundances of rock-forming elements (e.g., Fe, Si, Mg; Dorn et al. 2015). The observed relative abundances of $\mathrm{Fe} / \mathrm{Si}$ and $\mathrm{Mg} / \mathrm{Si}$ of planethosting stars have limited variability (Fig. 2). Here, we assume that the variability of stellar abundance ratios (Fe/Si and $\mathrm{Mg} / \mathrm{Si}$ ) is reflected in the bulk composition of the majority of superEarths. By using this assumption, we can calculate possible interior end-members that account for the anticipated variability of super-Earth structures and compositions. Furthermore, thermal states of super-Earths are expected to be highly variable, since observed planets have different ages. However, the thermal states of exoplanets are extremely difficult to constrain by observations. We thus use theoretical considerations to account for reasonable ranges of thermal parameters. On this basis, we investigate and compare how volcanic activity and outgassing is affected by the variability in structural, compositional, and thermal parameters.

Outgassing is dependent on the convection regime of a planet. The most likely convection regime of super-Earths is a matter of debate. Here, as well as briefly discussing other regimes, we focus on the stagnant-lid convection regime in order to fully investigate all relevant parameters. Furthermore, we restrict the volcanic outgassing to pure $\mathrm{CO}_{2}$, since it is one of the major outgassed volatiles (Gaillard \& Scaillet 2014). We focus on the accumulated amount of outgassed $\mathrm{CO}_{2}$ over the lifetime of $4.5 \mathrm{Gyr}$ in order to compare with Solar System planets. In addition, we discuss the time dependence of outgassing for a range of planet masses and for ages up to $10 \mathrm{Gyr}$ (see Sect. 6).

The paper is structured as follows. We first provide an introduction on convection regimes and previous studies. We then describe our methodology and results based on the large number of planet simulations. We provide scaling laws for parameters of first and second order influence and end with a discussion and conclusions.

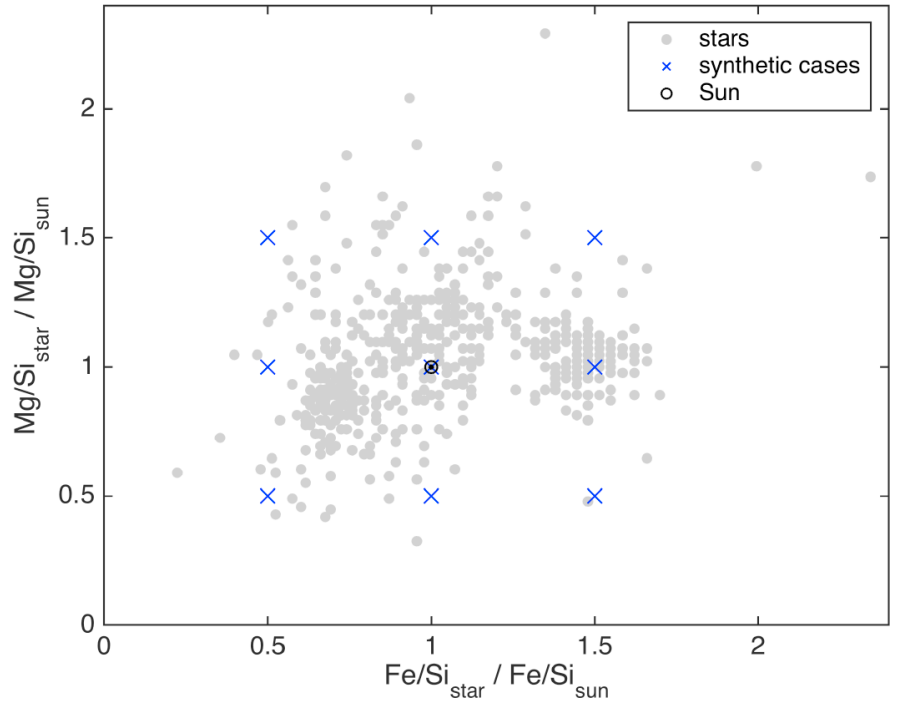

Fig. 2. Stellar abundances $\mathrm{Fe} / \mathrm{Si}_{\text {star }}$ and $\mathrm{Mg} / \mathrm{Si}_{\text {star }}$ for stars within $150 \mathrm{pc}$ based on the Hypatia catalog (Hinkel et al. 2014). Values are relative to solar estimates $\left(\mathrm{Fe} / \mathrm{Si}_{\text {Sun }}=1.69\right.$ and $\mathrm{Mg} / \mathrm{Si}_{\text {Sun }}=0.89$ based on Lodders (2003)). Blue crosses denote synthetic planetary bulk abundances used in our study $\left(\mathrm{Fe} / \mathrm{Si}_{\text {bulk }}=\{0.5,1 ., 1.5\} \times \mathrm{Fe} / \mathrm{Si}_{\text {Sun }}\right.$ and $\left.\mathrm{Mg} / \mathrm{Si}_{\text {bulk }}=\{0.5,1 ., 1.5\} \times \mathrm{Mg} / \mathrm{Si}_{\text {Sun }}\right)$

\section{Background}

\subsection{Convection regimes}

For a rocky planet there are commonly three possible convection regimes considered in geodynamics: mobile lid (potentially resembling plate tectonics; Mallard et al. 2016), stagnant-lid (Solomatov 1995) and episodic regime (Moresi \& Solomatov 1998; Stein et al. 2004). Yet, new convection regimes based on thermo-compositional convection are being reported (Moore \& Webb 2013; Gerya 2014; Sizova et al. 2015; Fischer \& Gerya 2016; Lourenço et al. 2016; Rozel et al. 2017). 
The stagnant-lid case is characterized by a very rigid lithosphere, which naturally occurs when lithospheric deformation is incapable of triggering mechanisms for localizing shear and weakening the high-viscosity lithosphere. In this case, the resistance of rocks to deform in the presence of stress, that is, the viscosity, is high. If no other rheological mechanism is included, the lithosphere is so viscous that in cannot be recycled in the deep mantle (Solomatov 1995) and deformation only occurs in the sublithospheric mantle. In this case, outgassing is possible by eruption of melt. Mercury, Mars, and the Earth's moon are examples of stagnant-lid regimes, where very intense craterization provides evidence that the lithosphere has not been entrained in the deep mantle for billions of years.

The lithosphere of the Earth does extensively deform due to several complex mechanisms (Kohlstedt et al. 1995): brittle failure (Byerlee 1978), evolution of microstructures at plate boundaries (Burov 2007), rock hydration-weakening and associated phase transitions (Mackwell et al. 1998; Schwartz et al. 2001), magmatism (Marsh 2010), and so on. Due to a combination of all these processes, deformations of the lithosphere can result in a mobile-lid regime (i.e., plate tectonics) in which the lithosphere is constantly recycled in the mantle. This allows greenhouse gases (e.g., $\mathrm{CO}_{2}, \mathrm{H}_{2} \mathrm{O}$ ) to cycle between mantle and atmosphere reservoirs by volcanism and subduction of carbonate sediments which result from weathering and erosion of surface rocks.

If mantle driving forces do not exceed lithospheric yield strength, the lithosphere slowly thickens and stresses grow until lithosphere deformation suddenly occurs through a catastrophic event during which the entire lithosphere sinks in the mantle (Fowler 1985; Reese et al. 1998) and outgassing is efficient (Gillmann \& Tackley 2014). In this so-called episodic regime, lithospheric growth and catastrophic resurfacing events happen episodically (Moresi \& Solomatov 1998; Stein et al. 2004). Venus might experience similar dynamics (Strom et al. 1994).

Likelihood of convection regimes. Determining the likelihood of convection regimes for super-Earths is still a very challenging problem in geodynamics. Many interdependent physical parameters are suspected to have a major effect on the dynamics of the lithosphere, which controls the global behavior of planetary mantles. The strikingly different regime behaviors of Earth and Venus indicate that other parameters besides planet mass and size are determining factors. The difference in solar incident fluxes is often used to explain their respective convection regimes, however, potential key parameters include rock hydration, thermal state, viscosity, melt fraction, compositional heterogeneities and grain size distributions. Heavy numerical implementations and computational resources are required to test these parameters in order to obtain robust scaling laws for the likelihood of different convection regimes.

After the discovery of the first exoplanets, different studies estimate the likelihood of plate tectonics with increasing planet mass and conclude increasing (Valencia et al. 2007a; Papuc \& Davies 2008; Valencia \& O'Connell 2009) and decreasing trends (Kite et al. 2009). Furthermore, effects of rock hydration (Korenaga 2010) and thermal states (internal heating versus basal heating and initial temperatures) (Van Heck \& Tackley 2011; Noack \& Breuer 2014), as well as complex rheologies and the pressure-dependence of many physical quantities (Tackley et al. 2013) can have first-order influences. Overall, the likelihood of different convection regimes for superEarths is ongoing research. Here, we focus on the stagnant-lid regime only.

\subsection{Previous studies}

In the following, we highlight a few principle studies that investigated outgassing on stagnant-lid planets. Kite et al. (2009) predict that stagnant-lid exoplanets have high melting rates even for massive super-Earth planets, but their model did not account for the fact that melt may be denser than surrounding solid mantle material at specific depths, leading to gravitationally stable melt, thus hindering surface volcanism and outgassing. They considered a purely temperature-dependent viscosity, which is expected to overestimate the mantle convective velocities, and therefore lead to increased melting rates.

Vilella \& Kaminski (2017) derived improved scaling laws for planets for variable convection strength and predict the thermal evolution and melt occurrence on Earth-like exoplanets. They propose that the occurrence of melting decreases with age and planetary radius. Large planets would only show melting early on in their evolution. This study also considers gravitationally stable melt.

Noack et al. (2014) investigated the outgassing efficiency for planets of variable core sizes and fixed Earth-like composition and size. Outgassing is strongly reduced for large core radius fractions $\left(>0.7 R_{\oplus}\right)$ due to the larger pressure gradient in the lithosphere. However, how likely such large core radius fractions are among super-Earths requires further research. While varying Earth-like planets to masses of up to $10 M_{\oplus}$ assuming magnesium-silicate mantles and different core-mass fractions, Noack et al. (2017) found that outgassing is limited to planets below 4-7 $M_{\oplus}$ (depending on other parameter assumptions).

Our study differs in several respects to the previous study of Noack et al. (2017):

- we test an extensive range of parameters for their influence on mantle outgassing, including planet mass, radiogenic heating, initial mantle temperature, initial lithosphere thickness, mantle composition in terms of $\mathrm{Mg} / \mathrm{Si}$ and $\mathrm{Fe} / \mathrm{Si}$, viscosity, density-cross-over pressure, and effects of hydration;

- the range of tested parameters reflects our anticipated variability of the majority of exoplanet interiors;

- our planet interior model allows for general mantle compositions in the $\mathrm{FeO}-\mathrm{SiO}-\mathrm{MgO}$ system;

- we quantify the influence of individual parameters on outgassing by providing a scaling law.

\section{Methodology}

\subsection{Calculation of interior end-members}

The first part of this study concerns the calculation of interiors that cover the anticipated variability of super-Earths. We calculate those interiors given commonly observed ranges of astrophysical data and theoretical prior considerations. The astrophysical data include planetary mass and radius, stellar bulk abundances, and associated uncertainties (listed below). Chosen data uncertainties compare to high data quality. For a specific super-Earth case, we use the probabilistic method of Dorn et al. (2015) to calculate the possible range of interiors. From this range, we identify those interiors of minimum and maximum core size that fit data within 1- $\sigma$ uncertainty. These represent the extracted end-members, which are input to the convection model. The extracted models provide profiles for temperature, density, thermal expansion coefficient, thermal heat capacity, thermal conductivity, gravity, and pressure. We provide more details on data and interior model in the following and refer to Dorn et al. (2015) for more details on the probabilistic method. 
Table 1. Summary of planetary mass and radius data.

\begin{tabular}{cc}
\hline \hline$M_{\mathrm{p}} / M_{\oplus}$ & $R_{\mathrm{p}} / R_{\oplus}$ \\
\hline 1. & 1. \\
1.5 & 1.1 \\
2. & 1.2 \\
2.5 & 1.28 \\
3. & 1.33 \\
4. & 1.44 \\
5.5 & 1.52 \\
6.6 & 1.6 \\
7.7 & 1.69 \\
8.8 & 1.74 \\
\hline
\end{tabular}

Notes. Uncertainties on mass and radius are $10 \%$ and 5\%, respectively.

Table 2. Prior ranges.

\begin{tabular}{lll}
\hline \hline Parameter & Prior range & Distribution \\
\hline$r_{\text {core }}$ & $(0.01-1) r_{\text {core }+ \text { mantle }}$ & Uniform in $r_{\text {core }}^{3}$ \\
$r_{\text {core+mantle }}$ & $(0.01-1) R_{\mathrm{p}}$ & Uniform in $r_{\text {core+mantle }}^{3}$ \\
$\mathrm{Fe} / \mathrm{Si}_{\text {mantle }}$ & $0-\mathrm{Fe} / \mathrm{Si}_{\text {bulk }}$ & Uniform \\
$\mathrm{Mg} / \mathrm{Si}_{\text {mantle }}$ & $\mathrm{Mg} / \mathrm{Si}_{\text {bulk }}$ & Gaussian \\
\hline
\end{tabular}

\subsubsection{Data}

The considered astrophysical data comprise the following, which are listed in Table 1 and illustrated in Figs. 1 and 2:

- planetary mass $M_{\mathrm{p}}$ (Table 1 , uncertainty is fixed to $10 \%$ );

- planetary radius $R_{\mathrm{p}}$ (Table 1 , uncertainty is fixed to $5 \%$ );

- bulk abundance $\mathrm{Fe} / \mathrm{Si}_{\text {bulk }}\left(\mathrm{Fe} / \mathrm{Si}_{\text {bulk }}=\{0.5,1 ., 1.5\} \times\right.$ $\mathrm{Fe} / \mathrm{Si}_{\text {Sun }}$, see Fig. 2, uncertainty is fixed to $20 \%$ );

- bulk abundance $\mathrm{Mg} / \mathrm{Si}_{\text {bulk }}\left(\mathrm{Mg} / \mathrm{Si}_{\text {bulk }}=\{0.5,1 ., 1.5\} \times\right.$ $\mathrm{Mg} / \mathrm{Si}_{\text {Sun }}$, see Fig. 2, uncertainty is fixed to $20 \%$ );

- surface temperature is set to $280 \mathrm{~K}$ for all cases.

Masses and radii are chosen such that they follow the massradius relationship of Earth-like interiors (Valencia et al. 2007b).

\subsubsection{Interior model}

Our planet interior model consists of a layered sphere with an iron core surrounded by a silicate mantle. We allow for variable mantle composition and thicknesses of core and mantle. For the mantle composition, we use the FMS model chemical system that comprises the oxides $\mathrm{FeO}-\mathrm{MgO}-\mathrm{SiO}_{2}$. Thus the interior parameters comprise:

- core size $r_{\text {core }}$

- size of core and mantle $r_{\text {core+mantle }}$;

$-\mathrm{Fe} / \mathrm{Si}_{\text {mantle }}$;

- $\mathrm{Mg} / \mathrm{Si}_{\text {mantle }}$.

The prior distributions for the model parameters are stated in Table 2 and are similar to those in Dorn et al. (2015, 2017).

We calculate the interiors using self-consistent thermodynamics for core and mantle. For the core we use the equation of state (EoS) fit of iron in the hexagonal close-packed (hcp) structure provided by Bouchet et al. (2013) on ab initio molecular dynamics simulations. For the silicate mantle, we compute equilibrium mineralogy and density as a function of pressure, temperature, and bulk composition by minimizing Gibbs free energy (Connolly 2009). We assume an adiabatic temperature profile for core and mantle.
The interior model is used to calculate interior end-members for super-Earths, that are subsequently used as input to the convection model in order to study melting and outgassing.

\subsection{Convection and melting model}

The employed convection and melting model is described in detail by Noack et al. (2017), but briefly outlined in the following.

We model convection in a compressible mantle in the 2D spherical annulus geometry (Hernlund \& Tackley 2008). In order to describe compressible flow, we use the truncated anelastic liquid approximation (TALA). In this approximation, radial reference profiles are used together with calculated lateral variation fields for temperature, density, and pressure (e.g., Schubert et al. 2001; King et al. 2010; Noack et al. 2017). The reference profiles are those of temperature, density, gravity, and pressure, as well as material properties of thermal expansion coefficient, thermal heat capacity, and thermal conductivity. These profiles are provided by the extracted end-member interiors (see Sect. 3.1). Given the TALA formulation, the convection code solves the conservation equations for mass, momentum, and energy (King et al. 2010; Noack et al. 2017).

The convection behavior of the mantle depends on the rheological properties of the generally polycrystalline rocks. Here, we use rheology laws that were developed specifically for Earth's mantle. For pressures in the upper mantle, we use the diffusion law for dry olivine from Karato \& Wu (1993),

$\eta(T, p)=2.6 \times 10^{10} \exp \left(\frac{3 \times 10^{5}+6 \times 10^{3} p}{R T}\right)$,

using the universal gas constant $R$; for pressures in the lower mantle, we use those of perovskite (pv) and post-perovskite (ppv) as derived by Tackley et al. (2013),

$$
\begin{array}{ll}
\eta(T, p)=2.5 \times 10^{11} \exp \left(\frac{3.7 \times 10^{5}+3.65 \times 10^{3} \exp \left(\frac{-p}{200}\right) p}{R T}\right), & \text { for } \mathrm{pv} \\
\eta(T, p)=3.6 \times 10^{8} \exp \left(\frac{7.8 \times 10^{5}+1.7 \times 10^{3} \exp \left(\frac{-p}{1100}\right) p}{R T}\right), & \text { for } \mathrm{ppv}
\end{array}
$$

for pressure $p$ given in GPa and temperature $T$ in $\mathrm{K}$. Thereby, we neglect compositional effects on rheology. However, we do investigate the role of the viscosity on our outgassing results by adding a viscosity prefactor $\Delta_{\eta}$ which is set to 10 in case 9 and 1 in all other cases. For the rheology laws given above, we obtain a reference viscosity of $1.6 \times 10^{20}, 3 \times 10^{23}$, and $1 \times 10^{34} \mathrm{~Pa}$ $\mathrm{s}$ for olivine, perovskite and post-perovskite, respectively, at a reference temperature of $1600 \mathrm{~K}$ and zero GPa.

Melting is tracked at every time step in our simulations. Partial melting occurs where mantle temperature exceeds the solidus temperature. If the melt is gravitationally buoyant, we assume that melt should rise immediately to the surface and outgas. Instead of transporting the melt to the surface, we calculate the amount of $\mathrm{CO}_{2}$ that should be outgassed. The residue is consequently depleted in volatiles. We use the same parametrization as Noack et al. (2017) for outgassing processes (Table 3), i.e., if melting occurs at pressures below the so-called density cross-over pressure $\left(P_{\text {cross-over }}\right)$, the melt with initially $1000 \mathrm{ppm}$ of $\mathrm{CO}_{2}$ rises to the surface and depletes by $10 \%$ in volatiles. Depending on mantle mixing and the occurrence of partial melting, this process can happen repeatedly, however, maximum mantle depletion $d_{\max }$ is set to $30 \%$ (volumetric fraction). Mantle depletion is thus directly linked to the amount of outgassed volatiles. To trace the volatile depletion in the mantle, we use a particle-in-cell approach. 


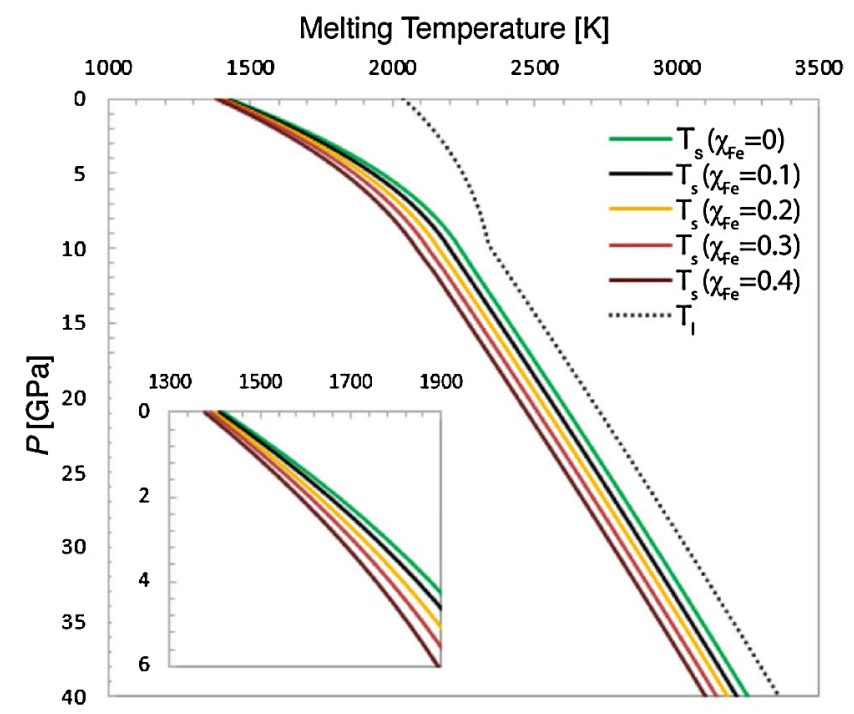

Fig. 3. Earth-like solidus ( $T_{\mathrm{s}}$, black solid line) and liquidus $\left(T_{1}\right.$, black dotted line) in comparison with melting temperatures as a function of iron weight fraction of the mantle $\left(\mathcal{X}_{\mathrm{Fe}}\right)$.

The amount of outgassing can be affected by mantle composition, because melting temperatures depend on rock composition (Kiefer et al. 2015). Based on the laboratory studies summarized in Kiefer et al. (2015) and Hirschmann (2000), we derive an iron-dependent melting law for low pressures. This is an addition to the usual solidus temperatures. For pressures above $12 \mathrm{GPa}$, the iron influence on the melting temperature is assumed to be pressure-independent:

$$
\begin{array}{ll}
\Delta_{T_{\mathrm{s}, \mathrm{Fe}}}=\left(102+64.1 p-3.62 p^{2}\right) \cdot\left(0.1-\mathcal{X}_{\mathrm{Fe}}\right), & \text { if } p \leq 12, \\
\Delta_{T_{\mathrm{s}, \mathrm{Fe}}}=360 \cdot\left(0.1-\mathcal{X}_{\mathrm{Fe}}\right), & \text { else. }
\end{array}
$$

The iron content $\mathcal{X}_{F e}$ is given in mass fraction and the pressure $p$ in GPa. The melting temperature for iron contents between 0 and 0.4 is depicted in Fig. 3.

For some test cases (10 and 11), we account for hydrated rock and use a wet solidus formulation taken from Katz et al. (2003) by assuming an initial amount of $500 \mathrm{wt}-\mathrm{ppm}$ water in all mantle rocks. The influence of water on the solidus is

$\Delta_{T_{\mathrm{s}, \mathrm{H} 2 \mathrm{O}}}=-43 X_{\mathrm{H} 2 \mathrm{O}}^{0.75}$,

where water content here is in wt-\%. Due to partial melting, water partitions into the melt for small melting degrees, and the residual material is set as dehydrated for melting depletion above $5 \mathrm{wt}-\%$. The melting solidus and liquidus temperatures for Earthlike mantle iron content $T_{\mathrm{s}}$ and $T_{1}$ are taken from Hirschmann (2000). The effective solidus temperature is then calculated as

$T_{\mathrm{s}, \mathrm{eff}}=T_{\mathrm{s}}+\Delta_{T_{\mathrm{s}, \mathrm{Fe}}}+\Delta_{T_{\mathrm{s}, \mathrm{H} 2 \mathrm{O}}}$.

Due to lack of experimental data, a more detailed treatment of the influence of composition on melting temperature is not justified for our study.

Initial temperatures in the mantle are cut if they lie above the solidus temperature, to avoid initial melting induced purely by the initial setup of the mantle. However, to be able to compare the simulations with a wet and dry solidus, for our wet mantle cases, we cut the initial mantle temperatures only if they lie above the dry solidus temperature.

We model the thermal evolution of all model planets over time. The initial temperature profile is calculated adiabatically starting from an initial upper mantle temperature $T_{\text {init,mantle, }}$ which is set beneath the lithosphere with an initial thickness of $T_{\text {init,mantle }}$. For most cases, we treat the core as isolated from the mantle, which means that the mantle temperatures evolve solely depending on the heat flux through the lithosphere, radioactive heat sources in the mantle, and latent heat consumption by melting. No heat flux from the core into the mantle is considered. In case 7, instead, we assume at the core mantle boundary an initial temperature difference between mantle and core $\Delta T_{\mathrm{cmb}}$ scaled with planet mass (Stixrude 2014). For this test case, the core cools with time and adds an additional heat source for the mantle. Radioactive heat sources vary between the different cases, from 0.5 to 1.5 times the Earth-like initial amount of heat, and decay over time (see Table 3). For Earth-like initial mass concentration of radiogenic elements, we assume $c_{\mathrm{U}^{235}}=1.2 \times 10^{-8}, c_{\mathrm{U}^{238}}=4.0 \times 10^{-8}, c_{\mathrm{Th}^{232}}=9.9 \times 10^{-8}$, $c_{\mathrm{K}^{40}}=3.7 \times 10^{-7}$. At $4.5 \mathrm{Gyr}$, these mass concentrations are $c_{\mathrm{U}^{235}}=1.4 \times 10^{-10}, c_{\mathrm{U}^{238}}=2.0 \times 10^{-8}, c_{\mathrm{Th}^{232}}=7.9 \times 10^{-8}$, $c_{\mathrm{K}^{40}}=3.1 \times 10^{-8}$ (McDonough \& Sun 1995) and are summarized as $1 \mathrm{c}_{\mathrm{E}}$. The total radiogenic heat production rate at $0 \mathrm{Gyr}$ is $24.2 \mathrm{pW} \mathrm{kg}^{-1}$

\section{Results}

We compiled a set of 2340 super-Earth models, for which we study melting and outgassing. This set covers our anticipated diversity of super-Earths in terms of structural, compositional, and thermal parameters.

The set comprises super-Earths of ten different masses and radii (see Table 1) and nine different bulk composition constraints (see Fig. 2), six different thermal parameters that stem from different formation conditions, and seven other parameters relevant for melting and interior dynamics (see Table 3 ). This yields a total of $10 \times 9 \times 13=1170$ models. In addition, for each super-Earth model, we consider two interior end-members (i.e., the models with minimum and maximum core size that fit data constraints). Thus, we have a total of $1170 \times 2=2340$ superEarth models, for which we simulate outgassing over a lifetime of $4.5 \mathrm{Gyr}$ (see Sect. 6 for time-dependence of outgassing up to $10 \mathrm{Gyr}$ ). The reference case comprises $10 \times 9 \times 2=180$ models as shown in Fig. 4. In the following, we discuss the individual cases.

\subsection{Outgassing versus planet mass}

Mantle depletion decreases with larger planet mass $M_{\mathrm{p}}$ (Fig. 4). For the $1 M_{\oplus}$ planet, the mantle is almost completely depleted after $4.5 \mathrm{Gyr}$, whereas for planets of 2-4 $M_{\oplus}$, depletion is significantly reduced (see also Fig. 5). This is because at higher masses, the pressure gradient in the lithosphere increases and thereby reduces the depth (or pressure) range, where melting can occur and melt is buoyant. Also, an increasing pressure at the bottom of the lithosphere results in higher melting temperature. We note that the pressure gradient $\mathrm{d} p / \mathrm{d} z$ that is plotted in Fig. 4 and later is defined as bulk density $\rho_{\text {bulk }}$ multiplied by gravity $\left(\mathrm{d} p / \mathrm{d} z=g \cdot \rho_{\text {bulk }}\right)$ and thus $\mathrm{d} p / \mathrm{d} z \sim M_{\mathrm{p}}^{2} / R_{\mathrm{p}}^{5}$. Here, the considered super-Earths roughly follow $R_{\mathrm{p}}=M_{\mathrm{p}}^{0.26}$ (Valencia et al. 2007b), thus $\mathrm{d} p / \mathrm{d} z$ changes nearly linearly with planet mass.

The amount of outgassed volatiles is denoted in partial pressure $p_{\mathrm{CO}_{2}}$ in bar, which is the mass of outgassed $\mathrm{CO}_{2}\left(m_{\mathrm{CO}_{2}}\right)$ times gravity divided by surface area:

$p_{\mathrm{CO}_{2}}=m_{\mathrm{CO}_{2}} g / 4 \pi R_{\mathrm{p}}^{2}$. 
Table 3. Input parameters of considered test cases.

\begin{tabular}{|c|c|c|c|c|c|c|c|}
\hline Parameter & Reference & Case 2 & Case 3 & Case 4 & Case 5 & Case 6 & Case 7 \\
\hline$Q_{\mathrm{rad}}$ & $1 c_{E}$ & $1.5 c_{E}$ & $\mathbf{0 . 5} c_{\mathrm{E}}$ & $1 c_{E}$ & $1 c_{E}$ & $1 c_{E}$ & \\
\hline$T_{\text {init,mantle }}$ & $1800 \mathrm{~K}$ & $1800 \mathrm{~K}$ & $1800 \mathrm{~K}$ & $1600 \mathrm{~K}$ & $2000 \mathrm{~K}$ & $1800 \mathrm{~K}$ & $1800 \mathrm{~K}$ \\
\hline$D_{\text {init,lith }}$ & $100 \mathrm{~km}$ & $100 \mathrm{~km}$ & $100 \mathrm{~km}$ & $100 \mathrm{~km}$ & $100 \mathrm{~km}$ & 50 km & $100 \mathrm{~km}$ \\
\hline$\Delta T_{\mathrm{cmb}}$ at the $\mathrm{CMB}$ & $0 \mathrm{~K}$ & $0 \mathrm{~K}$ & $0 \mathrm{~K}$ & $0 \mathrm{~K}$ & $0 \mathrm{~K}$ & $0 \mathrm{~K}$ & $\Delta \mathbf{T}_{\mathrm{cmb}}\left(M_{\mathrm{p}} / M_{\oplus}\right)^{*}$ \\
\hline Radial grid resolution & $25 \mathrm{~km}$ & $25 \mathrm{~km}$ & $25 \mathrm{~km}$ & $25 \mathrm{~km}$ & $25 \mathrm{~km}$ & $25 \mathrm{~km}$ & $25 \mathrm{~km}$ \\
\hline Viscosity prefactor $\Delta_{\eta}$ & 1 & 1 & 1 & 1 & 1 & 1 & 1 \\
\hline Wet/dry solidus & dry & dry & dry & dry & dry & dry & dry \\
\hline$P_{\text {cross-over }}$ & $12 \mathrm{GPa}$ & $12 \mathrm{GPa}$ & $12 \mathrm{GPa}$ & $12 \mathrm{GPa}$ & $12 \mathrm{GPa}$ & $12 \mathrm{GPa}$ & $12 \mathrm{GPa}$ \\
\hline Surface temperature & \multicolumn{7}{|c|}{$280 \mathrm{~K}$} \\
\hline Particles per cell & \multicolumn{7}{|c|}{10} \\
\hline Latent heat & \multicolumn{7}{|c|}{$600 \mathrm{~kJ} \mathrm{~kg}^{-1}$} \\
\hline Max. mantle depletion $d_{\max }$ & \multicolumn{7}{|c|}{$30 \%$} \\
\hline Amount of $\mathrm{CO}_{2}$ in melt $f_{\mathrm{CO}_{2}}$ & \multicolumn{7}{|c|}{$1000 \mathrm{ppm}$} \\
\hline Extrusive volcanism $f_{\mathrm{ex}}$ & \multicolumn{7}{|c|}{$10 \%$} \\
\hline Time of evolution & \multicolumn{7}{|c|}{ 4.5 Gyr } \\
\hline
\end{tabular}

\begin{tabular}{|c|c|c|c|c|c|c|}
\hline Parameter & Case 8 & Case 9 & Case 10 & Case 11 & Case 12 & Case 13 \\
\hline$Q_{\mathrm{rad}}$ & $1 c_{E}$ & $1 c_{E}$ & $1 c_{E}$ & $1 c_{\mathrm{E}}$ & $1 c_{E}$ & $1 c_{E}$ \\
\hline$T_{\text {init,mantle }}$ & $1800 \mathrm{~K}$ & $1800 \mathrm{~K}$ & $1800 \mathrm{~K}$ & $1600 \mathrm{~K}$ & $1800 \mathrm{~K}$ & $1800 \mathrm{~K}$ \\
\hline$D_{\text {init,lith }}$ & $100 \mathrm{~km}$ & $100 \mathrm{~km}$ & $100 \mathrm{~km}$ & $100 \mathrm{~km}$ & $100 \mathrm{~km}$ & $100 \mathrm{~km}$ \\
\hline$\Delta T_{\mathrm{cmb}}$ at the $\mathrm{CMB}$ & $0 \mathrm{~K}$ & $0 \mathrm{~K}$ & $0 \mathrm{~K}$ & $0 \mathrm{~K}$ & $0 \mathrm{~K}$ & $0 \mathrm{~K}$ \\
\hline Radial grid resolution & $10 \mathrm{~km}$ & $25 \mathrm{~km}$ & $25 \mathrm{~km}$ & $25 \mathrm{~km}$ & $25 \mathrm{~km}$ & $25 \mathrm{~km}$ \\
\hline Viscosity prefactor $\Delta_{\eta}$ & 1 & 10 & 1 & 1 & 1 & 1 \\
\hline Wet/dry solidus & dry & dry & wet & wet & dry & dry \\
\hline$P_{\text {cross-over }}$ & $12 \mathrm{GPa}$ & $12 \mathrm{GPa}$ & $12 \mathrm{GPa}$ & $12 \mathrm{GPa}$ & 8 GPa & $16 \mathrm{GPa}$ \\
\hline Surface temperature & \multicolumn{6}{|c|}{$280 \mathrm{~K}$} \\
\hline Particles per cell & \multicolumn{6}{|c|}{10} \\
\hline Latent heat & \multicolumn{6}{|c|}{$600 \mathrm{~kJ} \mathrm{~kg}^{-1}$} \\
\hline Max. mantle depletion $d_{\max }$ & \multicolumn{6}{|c|}{$30 \%$} \\
\hline Amount of $\mathrm{CO}_{2}$ in melt $f_{\mathrm{CO}_{2}}$ & \multicolumn{6}{|c|}{$1000 \mathrm{ppm}$} \\
\hline Extrusive volcanism $f_{\mathrm{ex}}$ & \multicolumn{6}{|c|}{$10 \%$} \\
\hline Time of evolution & \multicolumn{6}{|c|}{$4.5 \mathrm{Gyr}$} \\
\hline
\end{tabular}

Notes. $Q_{\text {rad }}$ are amounts of radioactive heat sources, $T_{\text {init,mantle }}$ is the initial upper mantle temperature, $D_{\text {init,lith }}$ is the initial lithosphere thickness, $\Delta T_{\mathrm{cmb}}$ is the temperature jump at the core-mantle-boundary (CMB), and $P_{\text {cross-over }}$ is the density-cross-over pressure. $Q_{\text {rad }}$ is in units of $\mathrm{c}_{\mathrm{E}}$, that is, the Earth-like amounts of radioactive heat sources, and represents present-day values (McDonough \& Sun 1995), from which initial amounts 4.5 billions years ago are calculated. Bold values indicate a variation with respect to the reference case. ${ }^{*}$ The function $\Delta T_{\mathrm{cmb}}\left(M_{\mathrm{p}} / M_{\oplus}\right)=1400 \mathrm{~K}$ (p: $\left.M_{\mathrm{p}} / M_{\oplus}\right)^{3 / 4}$ is taken from Stixrude (2014).

The influence of planet mass on $p_{\mathrm{CO}_{2}}$ is shown in Fig. 4. In this case, the absolute amount of outgassed $\mathrm{CO}_{2}$ increases with planet mass, because the absolute volume of mantle material and thus the volume of melt is larger. This trend dominates outgassing at small masses $\left(1-2 M_{\oplus}\right)$.

In Fig. 5 (lower half of each subplot), we show viscosity fields for the reference case at four different masses. Phase transitions between perovskite (pv) and post-perovskite (ppv) in the mantle are visible where viscosity increases by $\sim 1-2$ orders of magnitude. At large masses, mantle viscosities become relatively uniform which is due to a self-regulatory process (Tackley et al. 2013). This process can be understood as follows. Viscosity increases with pressure which tends to decrease the convective vigor. However, this leads to a higher internal temperature of the mantle. Since viscosity is temperature-dependent, viscosities are consequently lowered back to a level where global scale convection occurs.

\subsection{Outgassing versus thermal state}

Besides planet mass, thermal parameters have first-order effects on depletion and outgassing. Figure 6 shows how much an increase in radioactive heat sources $Q_{\text {rad }}$ and initial upper mantle temperatures $T_{\text {init,mantle }}$ leads to enhanced depletion and outgassing. We note that $T_{\text {init,mantle }}$ is the initial temperature at the boundary between lithosphere and upper mantle.

We vary the amount of radioactive heat sources from 0.5 to 1.5 times the Earth-like values (cases 2 and 3 in Table 3) to cover largely the expected variability range based on galactic evolution models (Frank et al. 2014) with regard to stellar ages (Silva Aguirre et al. 2015). An increase in the amount of radioactive heat sources can significantly enlarge the depth range where melting occurs. Thereby it enlarges the mass range of super-Earths, where depletion and outgassing are efficient. For example, maximal depletion is observed up to $1 M_{\oplus}$ for 


\section{Dorn et al.: Outgassing on stagnant-lid super-Earths}
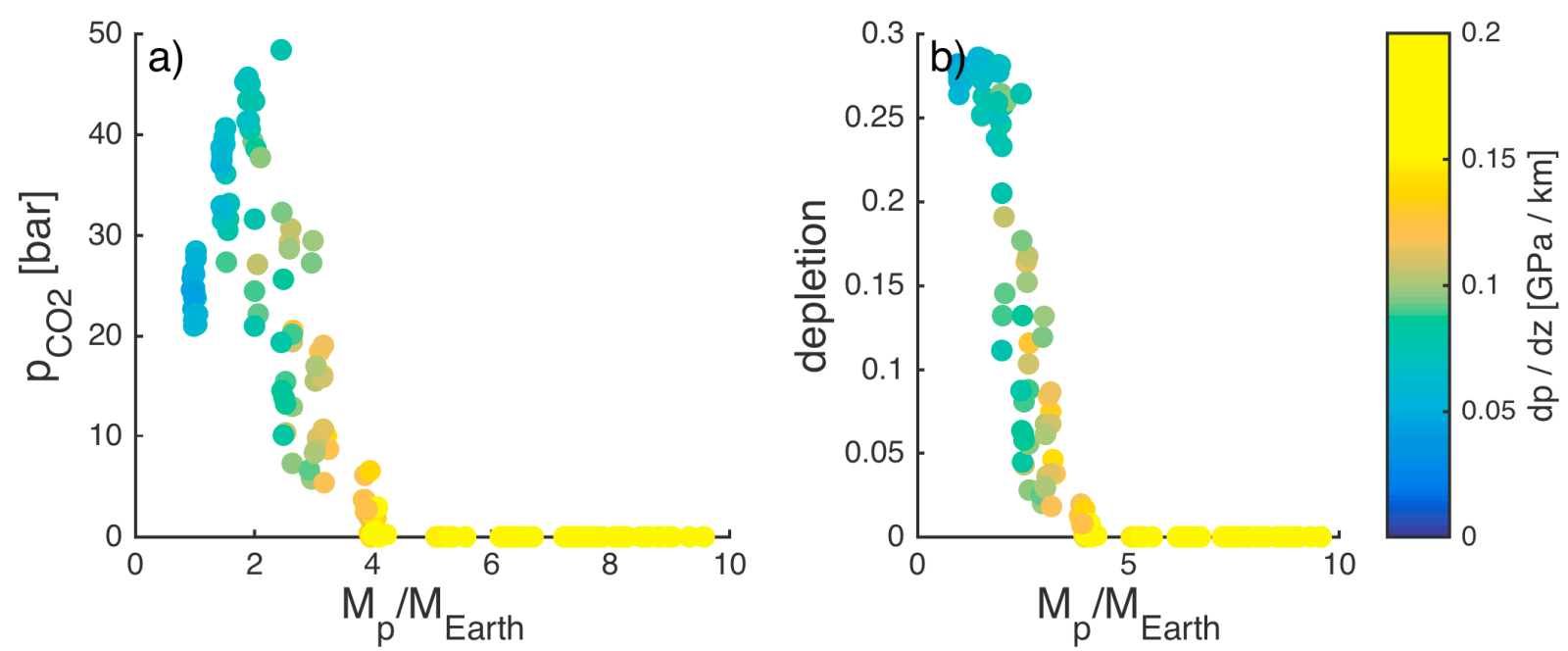

Fig. 4. Influence of planet mass $M_{\mathrm{p}}$ on $a$ ) outgassing and $b$ ) mantle depletion for the reference case (180 super-Earths shown) after 4.5 Gyr (see Table 3). The amount of outgassing of $\mathrm{CO}_{2}$ is denoted in terms of partial pressure $p_{\mathrm{CO}_{2}}$. Pressure gradient $\mathrm{d} p / \mathrm{d} z$ is shown in color. For plotting purposes, we saturated the color-scale at high values of $\mathrm{d} p / \mathrm{d} z$.

$Q_{\mathrm{rad}}=0.5 \mathrm{c}_{\mathrm{E}}$ and $3 M_{\oplus}$ for $Q_{\mathrm{rad}}=1.5 \mathrm{c}_{\mathrm{E}}$. This suggests that planets that formed early in the galactic history tend to be more depleted, since radiogenic heat sources were more abundant (Frank et al. 2014).

Similarly, an increase from 1600 to $2000 \mathrm{~K}$ for the $T_{\text {init,mantle }}$ (cases 4 and 5 in Table 3) extends the mass range of maximum mantle depletion from 1.5 to $2.5 M_{\oplus}$. The chosen range of variability in $T_{\text {init,mantle }}$ is based on the expected variation of upper mantle temperatures after the magma ocean state of a rocky planet, which is subject to the mantle composition and estimates for Earth-like compositions broadly cover 1600-2000 K (Herzberg et al. 2010; Jaupart et al. 2007).

In general, an increase in thermal parameters (i.e., $T_{\text {init,mantle, }}$ $Q_{\text {rad }}$ ) enables melting at shallower depths which partly outweighs for the pressure-limited melting depths at higher mass planets. Thereby, the mass range where depletion is most efficient can be extended up to $3 M_{\oplus}$. However, even on the initially hottest super-Earths (case 2 with $Q_{\mathrm{rad}}=1.5 \mathrm{c}_{\mathrm{E}}$ and case 5 with $T_{\text {init,mantle }}=2000 \mathrm{~K}$ ) depletion and outgassing only occur up to $7 M_{\oplus}$.

Similar effects are seen when considering super-heated cores that lead to a basally heated mantle. For Earth, how much heat flux there is at the CMB is still a matter of debate, and estimates suggest $20 \%$ of the total internal heating (Schubert et al. 2001). For test case 7, we use the mass-dependent powerlaw $\Delta T_{\mathrm{cmb}}\left(M_{\mathrm{p}} / M_{\oplus}\right)=1400 \mathrm{~K}\left(M / M_{\oplus}\right)^{3 / 4}$ by Stixrude $(2014)$ based on scaled thermal models. In general, the overall trend of outgassing on super-Earths is only weakly effected, however, the absolute amounts of outgassing can be significantly higher, especially for high-mass planets $\left(3-6 M_{\oplus}\right)$ as shown in Fig. 7.

\subsection{Outgassing versus interior structure}

The variation of core size and mantle composition seems to have a secondary influence on depletion and outgassing. In Fig. 5, we show planets of similar (solar-like) bulk composition, but with a different distribution of the bulk iron between core and mantle. The planets with large cores (right panels in Fig. 5) have little iron in the mantle, whereas small cores imply a higher iron mantle content. For the planets of 2 and $3 M_{\oplus}$, the interiors with higher iron mantle content and small cores seem less depleted. In this case, the mantle density is higher and leads to a higher pressure at the bottom of the lithosphere which reduces the depth range of buoyant melt production.

In Fig. 8 for a $2 M_{\oplus}$ planet, we show the effect on depletion and viscosity due to the variation of bulk composition in terms of $\mathrm{Fe} / \mathrm{Si}_{\text {bulk }}$ and $\mathrm{Mg} / \mathrm{Si}_{\text {bulk. }}$. Generally, the planets of high $\mathrm{Mg} / \mathrm{Si}_{\text {bulk }}$ and low $\mathrm{Fe} / \mathrm{Si}_{\text {bulk }}$ tend to be more depleted. Also, for a given bulk composition, the influence of the core size can result in larger or smaller mantle depletion. In Fig. $8 \mathrm{c}\left(\mathrm{Fe} / \mathrm{Si}_{\text {bulk }}=1.5 \mathrm{Fe} / \mathrm{Si}_{\text {sun }}\right.$ and $\left.\mathrm{Mg} / \mathrm{Si}_{\text {bulk }}=0.5 \mathrm{Mg} / \mathrm{Si}_{\text {sun }}\right)$, a larger core results in higher depletion (similar to Fig. 5), whereas for Fig. $8 \mathrm{a}\left(\mathrm{Fe} / \mathrm{Si}_{\text {bulk }}=\right.$ $0.5 \mathrm{Fe} / \mathrm{Si}_{\text {sun }}$ and $\mathrm{Mg} / \mathrm{Si}_{\text {bulk }}=0.5 \mathrm{Mg} / \mathrm{Si}_{\text {sun }}$, we see the opposite. In this case, the reduced mantle depletion can be explained by reduced melting due to an increase in melting temperature with less iron content.

The dependence of depletion and outgassing on core size and mantle composition is summarized in Fig. 9 for the reference case. For low-mass planets $\left(<2 M_{\oplus}\right)$, we see that increasing core size and decreasing mantle iron content leads to a decreased amount of outgassing. The amount of outgassed volatiles is limited by the absolute mantle volume, such that there is less outgassing for large cores. Mantle depletion in these cases is very efficient and weakly dependent on core size and mantle composition. Furthermore, influences of core size and mantle composition seem to become insignificant in the case of low radiogenic heating (case 3 in Table 3 ) (not shown).

For large-mass planets $\left(>2 M_{\oplus}\right)$, we see the opposite, in that larger core sizes and lower mantle iron contents result in higher amounts of outgassing and higher mantle depletion. In these cases, the melting region is relatively shallow and mostly within the lithosphere. High mantle iron contents imply a higher mantle density, which reduces melting by increasing the pressure at the bottom of the lithosphere. Melting is thus reduced to a shallower region. Even though a higher iron content lowers the melting temperature, which would imply enhanced depletion, the effect on mantle density is stronger.

We note that differences in mantle composition affect solidus temperatures as well as the reference profiles of temperature, density, gravity, and pressure, and also material properties of thermal expansion coefficient, thermal heat capacity, and 


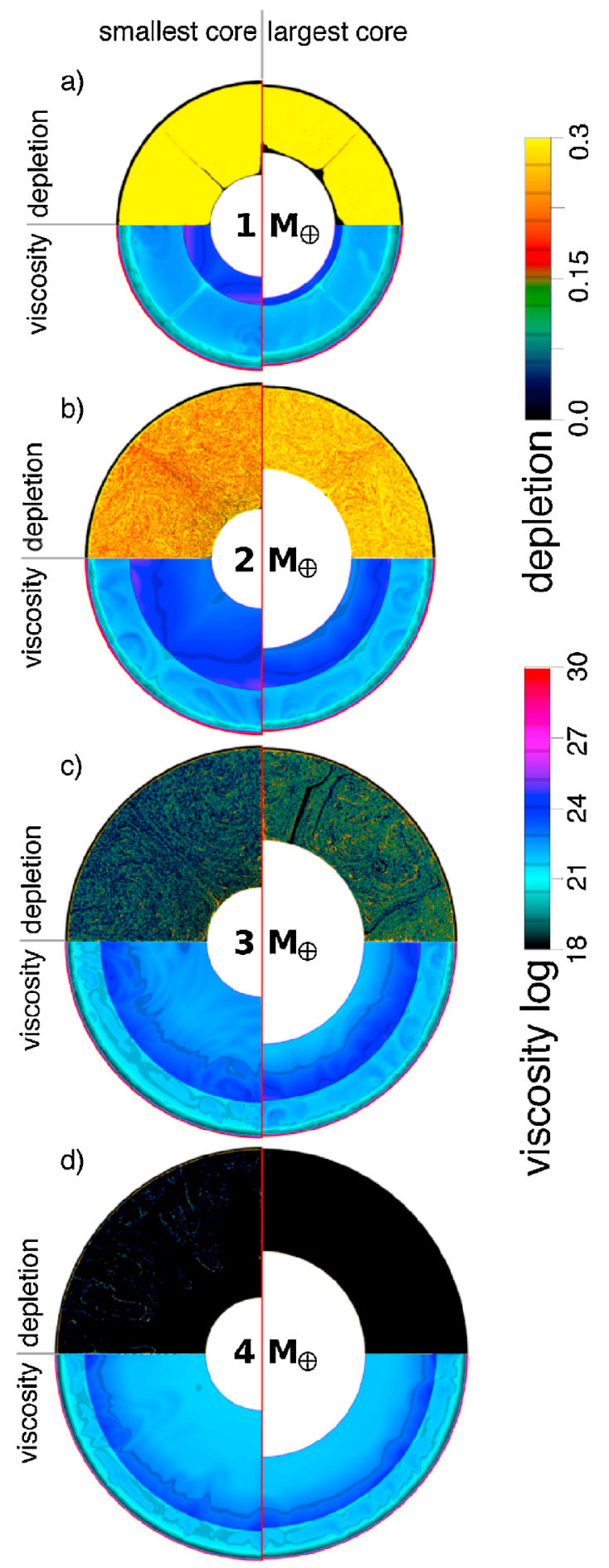

Fig. 5. Mantle depletion and viscosity for the reference case and for planets of $\left.\left.a) 1 M_{\oplus}, b\right) 2 M_{\oplus}, c\right) 3 M_{\oplus}$, and $\left.d\right) 4 M_{\oplus}$ after $4.5 \mathrm{Gyr}$. The left and right panels represent smallest and largest core sizes, respectively, that are in agreement with planet bulk abundances $\left(\mathrm{Fe} / \mathrm{Si}_{\text {bulk }}=\right.$ $1 \times \mathrm{Fe} / \mathrm{Si}_{\text {sun }}$ and $\left.\mathrm{Mg} / \mathrm{Si}_{\text {bulk }}=1 \times \mathrm{Mg} / \mathrm{Si}_{\text {sun }}\right)$.

thermal conductivity. Dependencies between composition and viscosity are not taken into account. Instead, we investigate effects of viscosity variations independent of mantle composition, which might overpredict the variability of depletion (see Sect. 4.4).

The initial lithosphere thickness (see case 6 in Table 3) only weakly influences volatile outgassing, which is depicted in
Fig. 10 for a thin $(50 \mathrm{~km})$ and thick $(100 \mathrm{~km})$ initial lithosphere thickness. As expected, a thinner initial lithosphere leads to slightly higher outgassing, since the initial melting depth extends deeper into the mantle.

\subsection{Outgassing versus viscosity}

Viscosity can significantly influence outgassing. An increased viscosity leads to less vigorous convection, thickens the lid and thereby reduces outgassing. The reduction of outgassing due to an increase in reference viscosity by a factor 10 is shown in Fig. 11. For all planet masses, outgassing is reduced, and the maximal outgassing efficiency is obtained for the smallest investigated mass of $1 M_{\oplus}$. A smaller reference viscosity (e.g., for increased amounts of iron, Zhao et al. 2009, or water, Hirth \& Kohlstedt 2003, in the mantle) would have the opposite effect.

At low masses $\left(<2 M_{\oplus}\right)$, where mantle depletion is most efficient and $p_{\mathrm{CO}_{2}}$ is at its maximum in the reference case, a decrease of viscosity would not further increase $p_{\mathrm{CO}_{2}}$. However, we expect significant influences at intermediate masses (2-4 $M_{\oplus}$ ). This intermediate mass range is also where the higher viscosity significantly reduces volcanic outgassing.

\subsection{Outgassing versus buoyant behavior of melt}

When melt occurs, its density contrast to the residue determines whether the melt migrates to the surface where it outgasses. The pressure up to which melt rises due to its buoyancy is parameterized by the density-cross-over pressure $P_{\text {cross-over }}$. Here we investigated the influence of $P_{\text {cross-over }}$ on the amount of outgassing. Reasonable ranges for $P_{\text {cross-over }}$ for anticipated variabilities of exoplanet mantle compositions are poorly understood. We test $P_{\text {cross-over }}$ being equal to 8,12 (reference), and $16 \mathrm{GPa}$, inspired by theoretical and empirical studies (Sakamaki et al. 2006; Bajgain et al. 2015). The resulting effect on the amount of outgassed $\mathrm{CO}_{2}$ is shown in Fig. 12. As expected, smaller values of $P_{\text {cross-over }}$ will lead to a reduced region where buoyant melt can exist and thus reduce outgassing (and vice versa).

At small planet masses $\left(<2 M_{\oplus}\right)$, where the depletion is most effective, an increase of $P_{\text {cross-over }}$ has marginal effects on outgassing. A significant influence on $p_{\mathrm{CO}_{2}}$ is only seen for the lower limit of $P_{\text {cross-over }}=8 \mathrm{GPa}$. At intermediate planet masses (2-4 $M_{\oplus}$ ), where outgassing is dominantly pressure-limited (see Sect. 4.1), the density-cross-over pressure can significantly alter the amount of outgassed $\mathrm{CO}_{2}$. For high planet masses above $5 M_{\oplus}$, volcanic outgassing is not effected by $P_{\text {cross-over. }}$.

\subsection{Outgassing versus hydration or mantle rock}

Little influence on volcanic outgassing is seen by accounting for hydration of rocks as illustrated in Fig. 13. We investigate the influence of a hydrated mantle (leading to a reduced solidus melting temperature; see Sect. 3.2) for two different initial mantle temperature profiles (cases 10 and 11). Water partitions very easily into the melt already for small fractions of partial melting. This results in rock being dehydrated very quickly and water being extracted during the early evolution. Therefore, the resulting amount of outgassed $\mathrm{CO}_{2}$ is only weakly effected by hydration of rocks. Over their lifetime, the amount of outgassed $\mathrm{CO}_{2}$ departs by less than 5 bars due to rock hydration for the majority of super-Earths. 

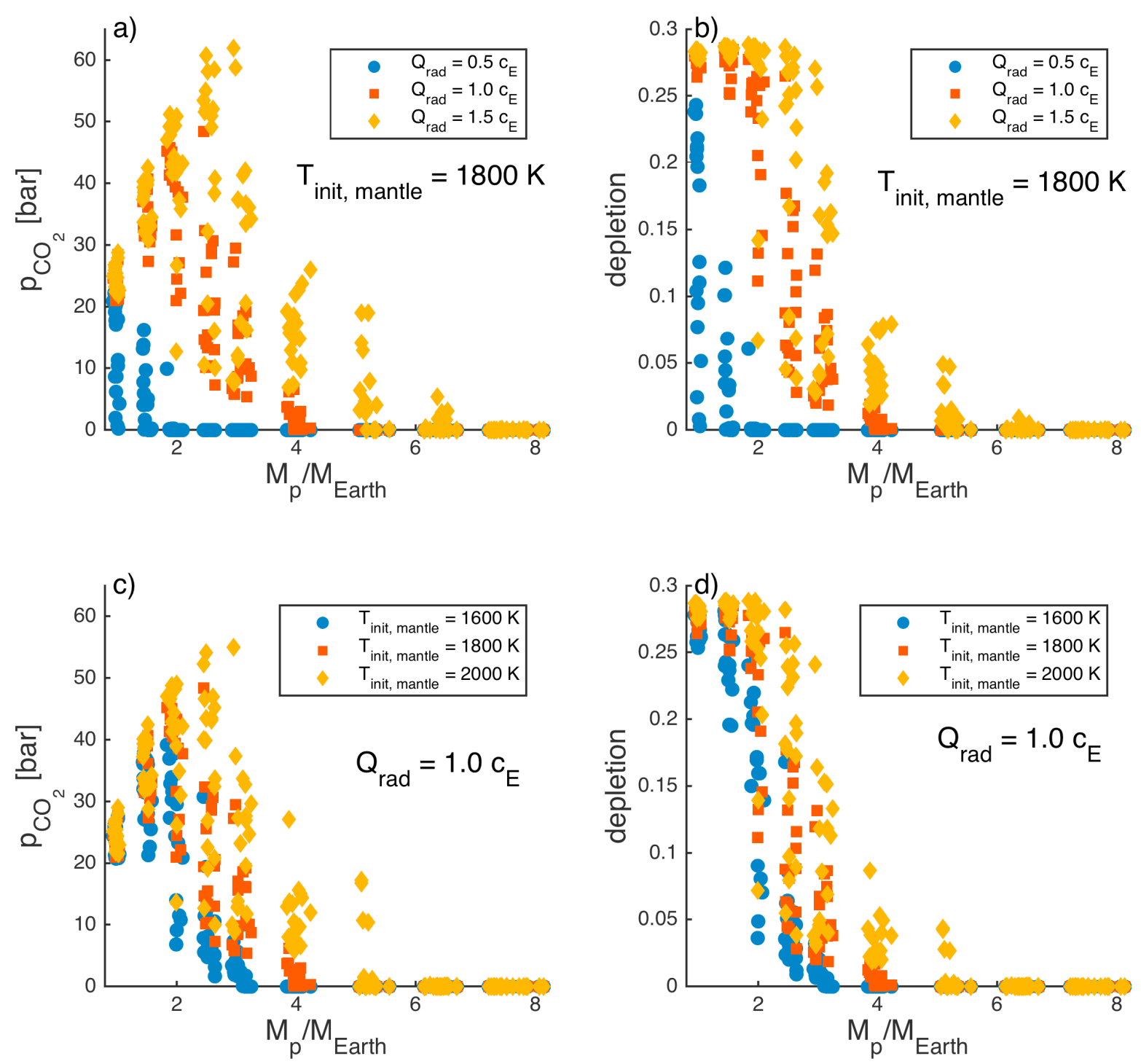

Fig. 6. Influence of $(a-b)$ radiogenic heating $Q_{\text {rad }}$ and $(c-d)$ initial mantle temperature $T_{\text {init,mantle }}$ on $(a, c)$ outgassing and $(b, d)$ mantle depletion. The amount of outgassing of $\mathrm{CO}_{2}$ is denoted in terms of partial pressure $p_{\mathrm{CO}_{2}}$. The cases 2-5 and the reference case are shown (Table 3).

\subsection{Resolution}

The radial resolution in the convection model is fixed to $25 \mathrm{~km}$ in the reference case and is set to a higher resolution of $10 \mathrm{~km}$ in case 8 (see Table 3 and Fig. 14). For small planet masses $\left(\leq 2 M_{\oplus}\right)$ with extended melting regions, the higher resolution marginally effects the outcome. However, for higher-mass planets $\left(2-5 M_{\oplus}\right)$ a higher resolution allows to better capture the extent of melting zones and thus outgassing estimates are on average $\sim 18 \%$ (6 bar) higher.

\section{Scaling of outgassing}

At an earlier stage of our study, we tried to describe the simulated outgassing using boundary layer theory only, however, we realized that the outgassing strongly depends on the internal temperature of the upper mantle, which is particularly poorly predicted by boundary layer theory. Here, we develop an empirical scaling law that uses boundary layer theory in parts to predict the above studied trends of mantle depletion and outgassing based on the large number of simulations. We focus on parameters that have first- and second-order effects on depletion and outgassing. Our proposed functional form for a scaling is underpinned by the following physical relationships, in which we introduce scaling parameters (i.e., $\alpha, \beta, \gamma, \zeta, \zeta_{1}, \zeta_{2}, \zeta_{3}, \zeta_{4}, \zeta_{5}, \nu, \theta, \kappa, \lambda, \mu$, $\xi, \psi, \omega)$.

Since melt depletion occurs at pressures below the cross-over pressure $\left(P_{\text {cross-over }}\right)$ and temperatures above solidus temperatures $T_{\mathrm{s}}$, we consider mantle depletion to be

$d=\beta \cdot\left(V_{\text {Pcross }} / V_{\text {mantle }}\right)^{\alpha} \cdot\left(T_{\text {eff }}-T_{\mathrm{s}}\right)^{\lambda}$,

where $V_{\text {Pcross }}$ is non-negative and is the part of the total mantle volume $V_{\text {mantle }}$ which is below the lid and in which pressures are below $P_{\text {cross-over }}$,

$V_{\text {Pcross }} \approx 4 / 3 \cdot \pi \cdot\left(\left(R_{\mathrm{p}}-\delta_{\mathrm{LAB}}\right)^{3}-\left(R_{\mathrm{p}}-\frac{P_{\text {cross-over }}}{\mathrm{d} p / \mathrm{d} z}\right)^{3}\right)$,

where $\mathrm{d} p / \mathrm{d} z$ is the pressure gradient. The depth $\delta_{\mathrm{LAB}}$ of the boundary between the rigid lithosphere and the ductile asthenosphere depends on viscosity $\eta$ and is approximated using 


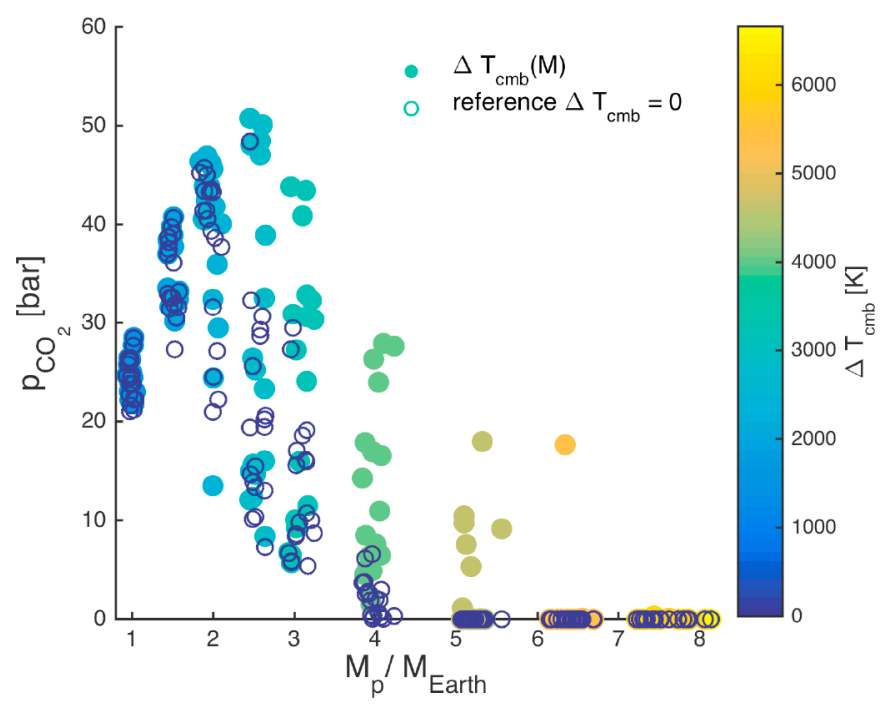

Fig. 7. Influence of a temperature increase at the core-mantle boundary $\Delta T_{\mathrm{cmb}}$ on outgassing. The amount of outgassing of $\mathrm{CO}_{2}$ is denoted in terms of partial pressure $p_{\mathrm{CO}_{2}}$. Case 7 and the reference case are shown (Table 3).

the asymptotic solutions of the Stokes equation (Reese et al. 1998):

$\delta_{\mathrm{LAB}}=\psi R_{\mathrm{p}} \Delta \eta^{0.2}$

We obtain a best-fit value for the exponent of 0.2 in Eq. (4), which is similar to the exponent derived for Newtonian convection from asymptotic boundary layer theory (Fowler 1985; Solomatov 1995; Reese et al. 1998) and numerical studies (Reese et al. 1999). The value is below the classical exponent of one third, derived for steady-state boundary layer theory (Solomatov 1995), since our simulations are timedependent and use a temperature-and pressure-dependent viscosity (Hüttig \& Breuer 2011). Combining Eqs. (3) and (4), we obtain

$$
\begin{aligned}
V_{\text {Pcross }} \approx & 4 / 3 \cdot \pi \cdot\left(R_{\mathrm{p}}^{3}\left(1-\psi \Delta_{\eta}^{0.2}\right)^{3}\right. \\
& \left.-\left(R_{\mathrm{p}}-\frac{P_{\text {cross-over }}}{\mathrm{d} p / \mathrm{d} z}\right)^{3}\right)
\end{aligned}
$$

The volume of the mantle is defined by

$V_{\text {mantle }}=4 / 3 \cdot \pi \cdot\left(R_{\mathrm{p}}^{3}-r_{\text {core }}^{3}\right)$.

The solidus temperature varies depending on the iron mass fraction, which is discussed in Sect. 3.2.

$T_{\mathrm{s}}=\zeta_{1}+\Delta_{T_{\mathrm{s}}}=\zeta_{1}+360 \cdot\left(0.1-\mathcal{X}_{\mathrm{Fe}}\right)$.

The effective mantle temperature $T_{\text {eff }}$ represents a time-averaged temperature, which we intend to use for the scaling. We assume that $T_{\text {eff }}$ departs linearly from a reference case depending on both the initial mantle temperature $T_{\text {init,mantle }}$ and the number of radiogenic heating sources, with $T_{\text {init,mantle }}=1800 \mathrm{~K}$ and $Q_{\text {rad }}=1 \mathrm{c}_{\mathrm{E}}$ being reference values.

$$
\begin{aligned}
T_{\mathrm{eff}}= & \zeta_{2}+\zeta_{3}\left(T_{\text {init,mantle }}-1800 \mathrm{~K}\right) \\
& +\zeta_{4}\left(Q_{\mathrm{rad}}-1 \mathrm{c}_{\mathrm{E}}\right)+\zeta_{5} \log \left(\Delta_{\eta}\right)
\end{aligned}
$$

Also, we account for the influence of $\mathrm{Mg} / \mathrm{Si}_{\text {bulk }}$ and core size $r_{\text {core }}$ on depletion, as discussed in Sect. 4.3. A linear influence of $\mathrm{Mg} / \mathrm{Si}_{\text {bulk }}$ on depletion is appropriate given our test models. We find that the influence of core size $\left(r_{\text {core }}\right)$ and mantle iron content $\left(X_{\mathrm{Fe}}\right)$ can better predict depletion (by $20 \%$ ), when a secondorder term is used that involves radiogenic heating sources. This is because for low-radiogenic heating (case 3 in Table 3), we do not observe significant influences of $r_{\text {core }}$ and $\mathcal{X}_{\mathrm{Fe}}$ on depletion.

On this basis and by combining the above equations as well as normalizing the linear scaling factors by the reference values, we finally obtain:

$$
\begin{aligned}
d_{\text {pred }}= & \max \left\{0, \min \left\{d_{\text {max }},\left(V_{\text {Pcross }}(\psi) / V_{\text {mantle }}\right)^{\alpha} \cdot \beta\right.\right. \\
& \times\left(1+\gamma \frac{\left(T_{\text {init,mantle }}-1800 \mathrm{~K}\right)}{1800 \mathrm{~K}}\right. \\
& +\zeta \frac{\left(Q_{\mathrm{rad}}-\mathrm{c}_{\mathrm{E}}\right)}{\mathrm{c}_{\mathrm{E}}} \\
& +\omega \log \left(\Delta_{\eta}\right) \\
& +\nu \frac{\mathrm{Mg} / \mathrm{Si}_{\mathrm{bulk}}}{\mathrm{Mg} / \mathrm{Si}_{\mathrm{Sun}}} \\
& -\theta \cdot \frac{\left(\mathcal{X}_{\mathrm{Fe}}-0.1\right)}{0.1} \cdot \frac{\left(Q_{\mathrm{rad}}-\mu \cdot \mathrm{c}_{\mathrm{E}}\right)}{\left(\mathrm{c}_{\mathrm{E}}-\mu \cdot \mathrm{c}_{\mathrm{E}}\right)} \\
& \left.\left.\left.+\kappa \frac{r_{\mathrm{core}}}{R_{\mathrm{p}}} \cdot \frac{\left(Q_{\mathrm{rad}}-\mu \cdot \mathrm{c}_{\mathrm{E}}\right)}{\left(\mathrm{c}_{\mathrm{E}}-\mu \cdot \mathrm{c}_{\mathrm{E}}\right)}\right)^{\lambda}\right\}\right\},
\end{aligned}
$$

where $V_{\text {Pcross }}(\psi)$ refers to Eq. (5). The scaling parameters (i.e., $\alpha, \beta, \gamma$, etc.) and their fitted values are listed in Table 4 . We use a nonlinear regression model (i.e., the fitnlm function of MAT$\mathrm{LAB}$ ) in order to determine the scaling parameters such that the root mean squared (RMS) error (L2-norm) of the difference between simulated $d$ and $d_{\text {pred }}$ is minimized. We find an RMS error of 0.028 . The fit and associated residuals between simulated and predicted depletion is depicted in Fig. 15. The quality of the fit is limited due to the statistical nature of the interior model selection that results in moderate scatter which Eq. (9) does not fully capture.

The amount of total outgassed $\mathrm{CO}_{2}$ in terms of partial pressure $\left(p_{\mathrm{CO}_{2}}\right)$ is proportional to the mass of outgassed $\mathrm{CO}_{2}$. The mass of $\mathrm{CO}_{2}$ depends on depletion $d$ and the amounts of outgassed volatiles in the mantle, which is constant in all cases (here: 1000 ppm, see Table 3).

$m_{\mathrm{CO}_{2}} \propto d \cdot V_{\text {mantle }}$,

and using a restatement of Newton's second law the pressure that corresponds to the mass of outgassed $\mathrm{CO}_{2}$ is described as

$p_{\mathrm{CO}_{2}}=\frac{m_{\mathrm{CO}_{2}} \cdot g}{4 \pi R_{\mathrm{p}}^{2}}$,

and thus the predicted $p_{\mathrm{CO}_{2}}$ in bar can be written as

$p_{\mathrm{CO}_{2}, \text { pred }}=\xi \cdot 10^{-5} \cdot \frac{d_{\text {pred }}\left(R_{\mathrm{p}}^{3}-r_{\text {core }}^{3}\right) G M_{\mathrm{p}}}{3 R_{\mathrm{p}}^{4}}$,

where $\xi$ is another scaling parameter, $G$ is the gravitational constant, and the factor $10^{-5}$ accounts for the conversion from SI-units to bar. While using the predicted mantle depletion $d_{\text {pred }}$ in the above equation, we do another nonlinear regression to 

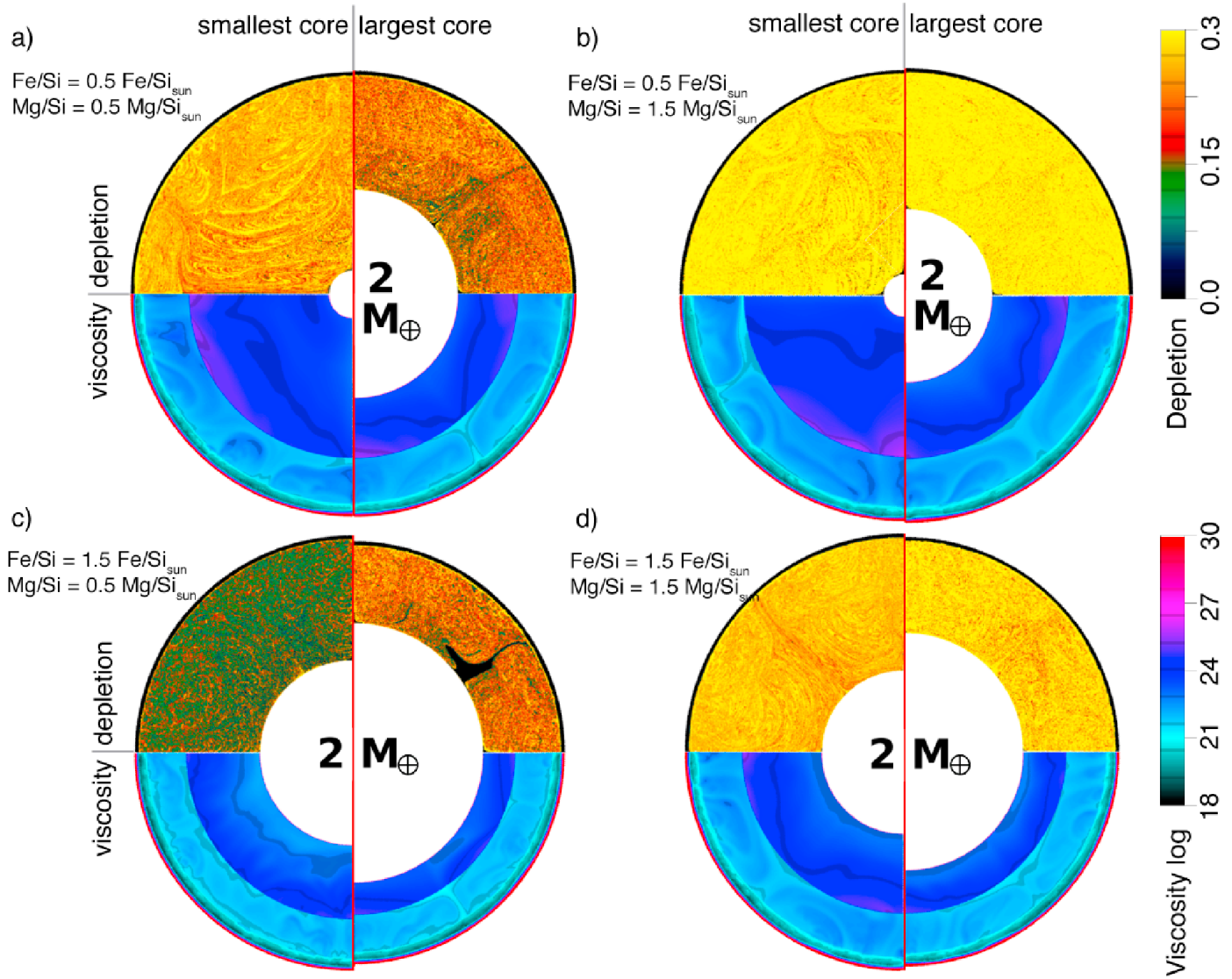

Fig. 8. Mantle depletion and viscosity for different planet bulk compositions after $4.5 \mathrm{Gyr}$. All shown planets have a mass of $2 M_{\oplus}$. The left and right panels represent smallest and largest core sizes, respectively, that are in agreement with planet bulk abundances $\left(\mathrm{Fe} / \mathrm{Si} \mathrm{bulk}_{\text {bu }}\right.$ and $\left.\mathrm{Mg} / \mathrm{Si} i_{\text {bulk }}\right)$. The reference case is shown (Table 3).

determine $\xi$ in order to best fit $p_{\mathrm{CO}_{2}}$ by $p_{\mathrm{CO}_{2}, \text { pred }}$. We expect $\xi$ to be on the order of the multiplication of $f_{\mathrm{ex}} \cdot f_{\mathrm{CO}_{2}} \cdot \bar{\rho}_{\text {mantle }}$ (see Table 3 ), which is approximately 0.5 for a mean mantle density $\bar{\rho}_{\text {mantle }}$ of $5000 \mathrm{~kg} \mathrm{~m}^{3}$. Indeed, our estimate for $\xi$ of 0.786 is on the same order. Figure 16 illustrates the quality of the fit for $p_{\mathrm{CO}_{2} \text {,pred, }}$, which is mostly limited by the residual scatter in $d_{\text {pred }}$ and has a RMS of 5.43 .

In addition to the proposed scaling laws, we extensively tried different functional forms, including nonlinear formulations and second order linear combinations of all parameters, but did not obtain significantly better fits.

Gas layer thicknesses. We demonstrated that the amount of outgassing is most efficient around $\sim 2 M_{\oplus}$, where the highest values of $p_{\mathrm{CO}_{2}}$ can be observed. In Fig. 17, we demonstrate how the distribution of $p_{\mathrm{CO}_{2}}$ would translate to gas layer thicknesses. We calculate the thicknesses $\Delta R_{\mathrm{CO}_{2}}$ using a scale height model similar to the model in Dorn et al. (2017):

$\Delta R_{\mathrm{CO}_{2}}=H \ln \left(\frac{p_{\mathrm{CO}_{2}}}{p_{\min }}\right)$

where $p_{\text {min }}$ is the pressure at which the atmosphere becomes opaque, which we simply fix to 1 mbar. The pressure scale height $H$ is calculated assuming a $\mathrm{CO}_{2}$ atmosphere (mean molecular weight of $44.01 \mathrm{~g} \mathrm{~mol}^{-1}$ ) and using a mean atmospheric temperature $T_{\text {atm }}$,

$H=\frac{T_{\mathrm{atm}} R^{*}}{g \cdot 44.01 \mathrm{gmol}^{-1}}$,
Table 4. Estimates of scaling parameter.

\begin{tabular}{llll}
\hline \hline Parameter & Estimate & \multicolumn{1}{c}{$\sigma$} & \multicolumn{1}{c}{$p$-value } \\
\hline \multicolumn{4}{c}{ (10 parameters, Eqs. (9) \& (12)): } \\
\hline$\alpha$ & 4.868 & 0.045 & 0 \\
$\beta$ & 45032.612 & $8 \times 10^{-7}$ & 0 \\
$\gamma$ & 2.50 & 0.20 & $2 \times 10^{-34}$ \\
$\zeta$ & 0.843 & 0.08 & $1 \times 10^{-27}$ \\
$\nu$ & 0.038 & 0.01 & $3 \times 10^{-7}$ \\
$\theta$ & 0.164 & 0.02 & $6 \times 10^{-16}$ \\
$\kappa$ & 0.486 & 0.06 & $2 \times 10^{-17}$ \\
$\lambda$ & 2.968 & 0.17 & $3 \times 10^{-62}$ \\
$\mu$ & 0.721 & 0.01 & 0 \\
$\xi$ & 0.786 & 0.02 & $5 \times 10^{-250}$ \\
$\psi$ & -0.002 & 0.0005 & 0.00021 \\
$\omega$ & -0.410 & 0.02 & $7 \times 10^{-69}$ \\
\hline
\end{tabular}

Notes. The standard deviation is denoted with $\sigma$, and the quality of fit with the $p$-value. We note that the significance of parameter estimates are only marginal in cases where $p$-values are larger than 0.05 .

where $g$ is surface gravity and $R^{*}$ is the universal gas constant (8.3144598 $\mathrm{J} \mathrm{mol}^{-1} \mathrm{~K}^{-1}$ ).

While $p_{\mathrm{CO}_{2}}$ first increases and then decreases with planet mass, the corresponding thicknesses $\Delta R_{\mathrm{CO}_{2}}$ always decrease with planet mass $M_{\mathrm{p}}$. This is because the scale height $H$ is inversely proportional to $g$, and thus $H \sim 1 / M_{\mathrm{p}}$. Our approximation of $\Delta R_{\mathrm{CO}_{2}}$ represents the thickness of the outgassed 

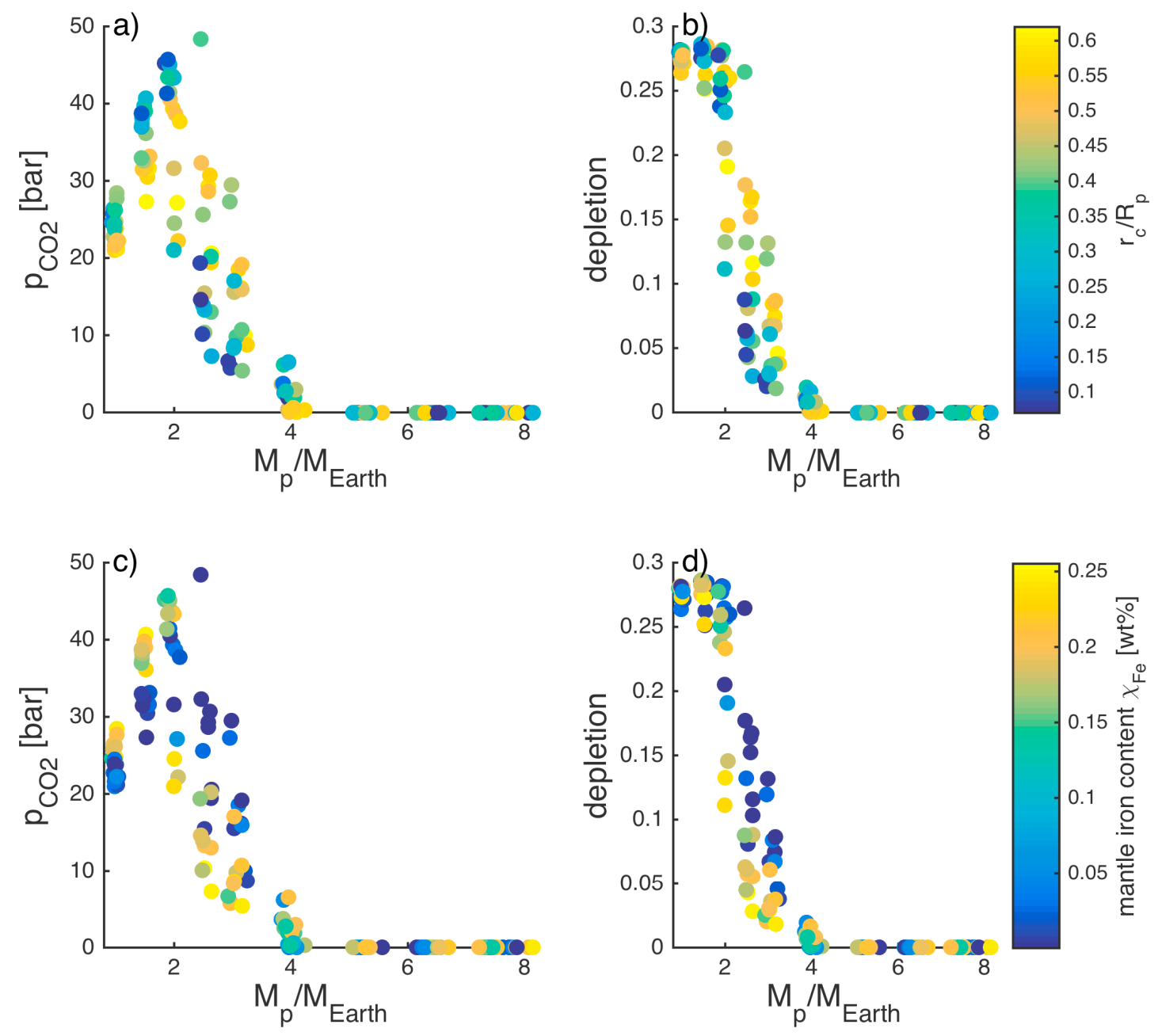

Fig. 9. Influence of $(a-b)$ core radius fraction $r_{\text {core }} / R_{\mathrm{p}}$ and $(c-d)$ mantle iron content $\mathcal{X}_{F e}$ on $(\mathrm{a}, \mathrm{c})$ outgassing and $(b, d)$ mantle depletion. The amount of outgassing of $\mathrm{CO}_{2}$ is denoted in terms of partial pressure $p_{\mathrm{CO}_{2}}$. The reference case is shown (Table 3 ).

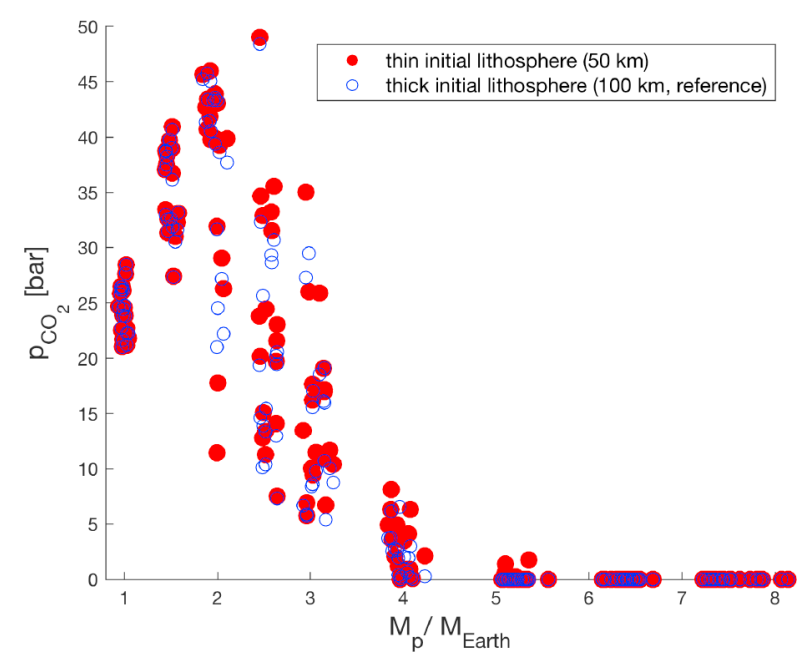

Fig. 10. Influence of the initial lithosphere thickness on outgassing. The amount of outgassing of $\mathrm{CO}_{2}$ is denoted in terms of partial pressure $p_{\mathrm{CO}_{2}}$. Case 6 and the reference case are shown (Table 3).

atmosphere, neglecting any primary or primordial atmosphere, chemical weathering, or atmospheric escape. We compare $\Delta R_{\mathrm{CO}_{2}}$ with independent atmospheric estimates for Venus and Mars and find good agreement (Fig. 17). Compared to our scaled estimates, the thicker atmosphere on Venus can be explained by

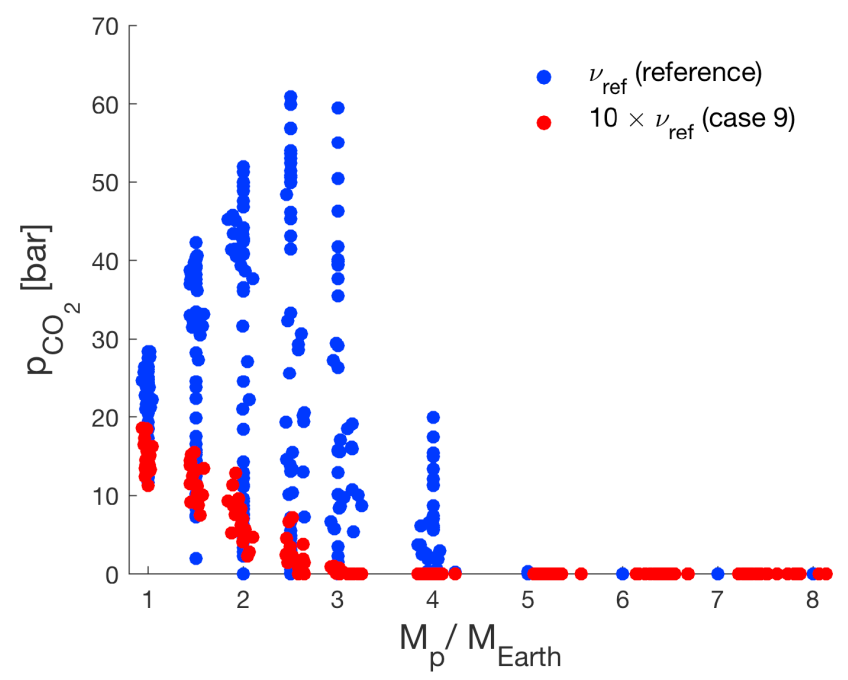

Fig. 11. Influence of reference viscosity on outgassing as a function of planet mass. Here, we compare the reference case to case 9 .

catastrophic outgassing events, whereas the thinner atmosphere on Mars can be explained by atmospheric erosion. We note that for both Venus and Mars, regassing of $\mathrm{CO}_{2}$ into the mantle is precluded, which is also due to the lack of liquid surface water and plate tectonics. 


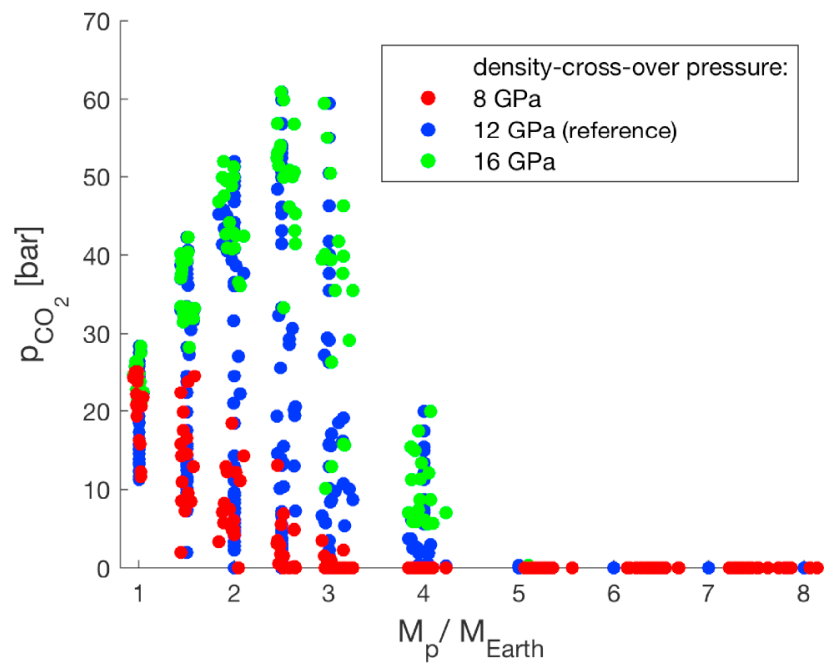

Fig. 12. Influence of density-cross-over pressure $P_{\text {cross-over }}$ (red, green, and blue dots) on the amount of outgassing as a function of planet mass. Reference case and cases 12 and 13 are shown.

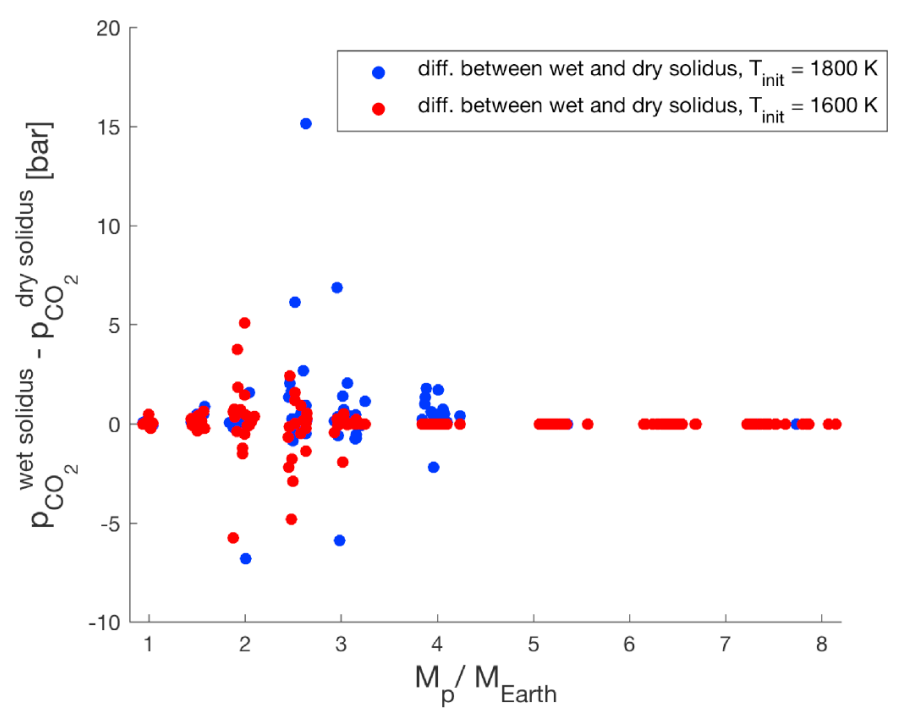

Fig. 13. Influence of rock hydration on outgassing: the difference in the amounts of outgassed $\mathrm{CO}_{2}$ between dry and wet solidus melting temperatures are plotted versus planet mass for two different initial mantle temperatures $T_{\text {init }}(1600 \mathrm{~K}$ and $1800 \mathrm{~K})$. The shown differences are comparisons between case 10 and the reference case (blue dots) as well as case 11 and 4 (red dots).

\section{Time-dependency}

Our empirical scaling law for depletion in Eq. (9) is not timedependent. Yet, Fig. 18 shows that we were able to nicely reproduce the time-dependence of depletion of our reference cases using a simple model based on boundary layer theory which we describe in the following.

In order to reproduce the temporal evolution of the depletion, we consider that the mantle is divided into three layers: the lithosphere, the $\mathrm{CO}_{2}$ producing region (from the bottom of the lithosphere to the cross-over depth) and the nondepleting mantle (everything below the cross-over depth). We consider that the lithosphere thickness is governed by the Rayleigh number (Ra), as indicated by boundary layer theory (Fowler 1985; Solomatov 1995; Reese et al. 1998). Since both the viscosity of the mantle and the melt fraction in the $\mathrm{CO}_{2}$-producing region

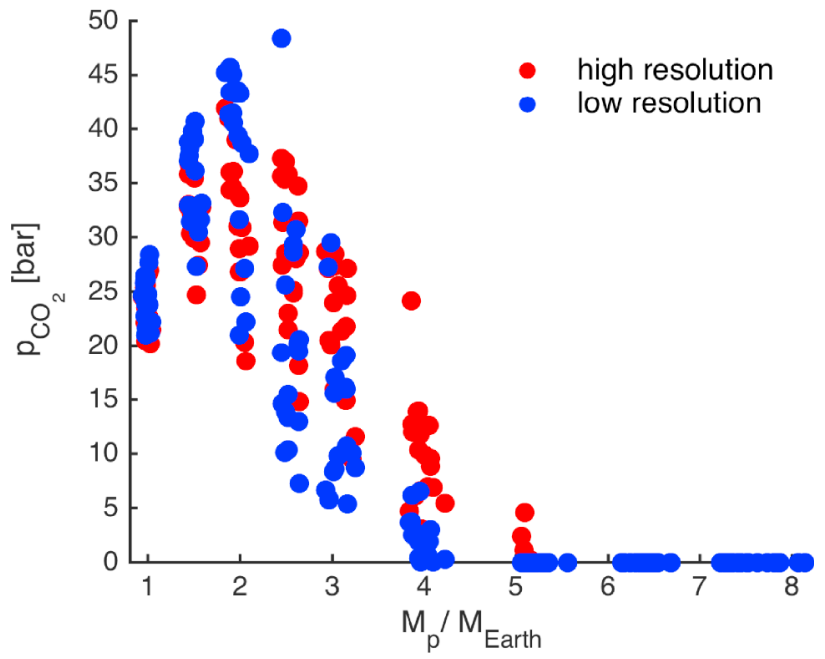

Fig. 14. Influence of model resolution on simulated outgassing. The amount of outgassing of $\mathrm{CO}_{2}$ is denoted in terms of partial pressure $p_{\mathrm{CO}_{2}}$. Case 8 and the reference case are shown (Table 3). Low-resolution (blue) refers to a radial resolution of $25 \mathrm{~km}$, whereas high-resolution (red) refers to a $10 \mathrm{~km}$ resolution.
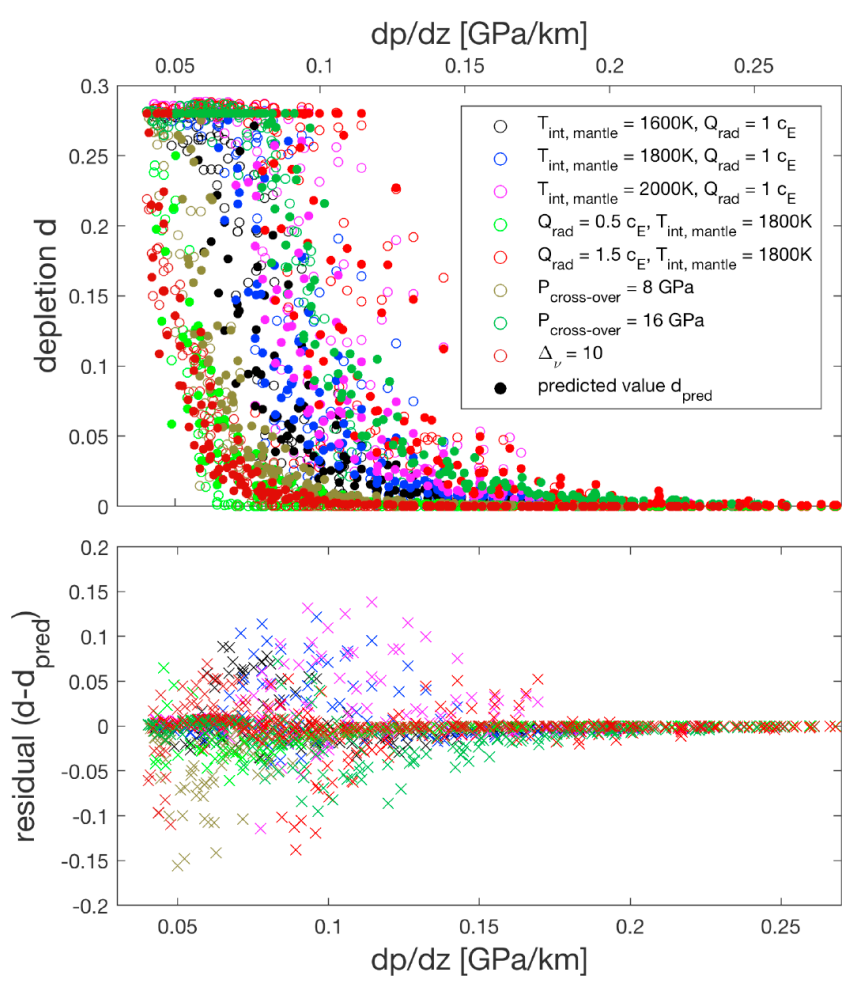

Fig. 15. Fit between simulated and predicted mantle depletion using Eq. (9) (upper panel) and corresponding residuals (lower panel).

are temperature-dependent, we carefully model the evolution of a reference temperature throughout time.

\subsection{Evolution of the temperature}

We numerically integrate the evolution of the temperature of the $\mathrm{CO}_{2}$-producing region using the simple form:

$T(t)=T_{0}+\int_{t^{\prime}=0}^{t} \frac{\partial T}{\partial t^{\prime}} \mathrm{d} t^{\prime}$. 

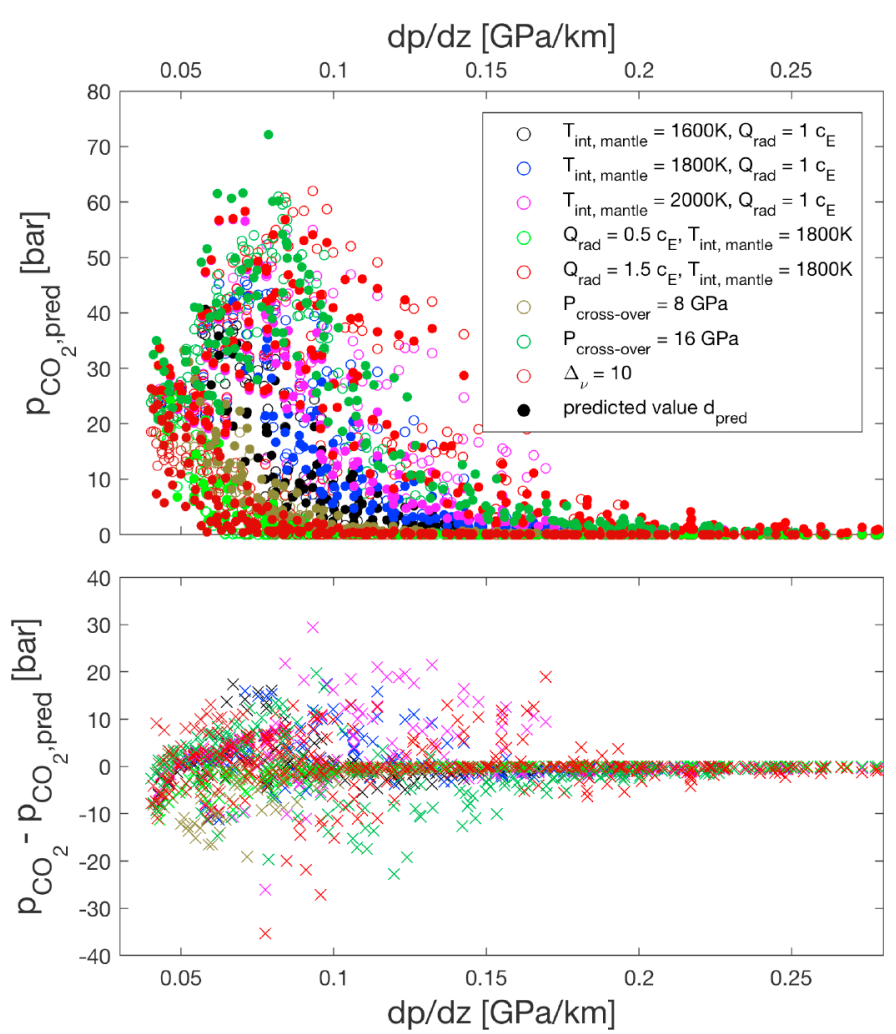

Fig. 16. Fit between simulated and predicted amounts of outgassed $\mathrm{CO}_{2}$ using Eqs. (9) and 12 (upper panel) and the corresponding residuals (lower panel).

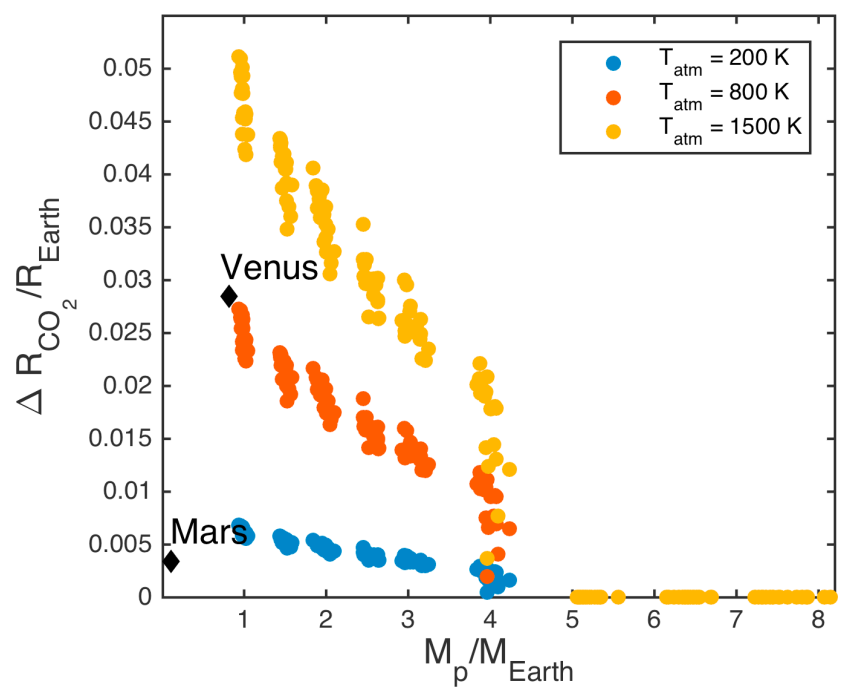

Fig. 17. Gas layer thicknesses corresponding to calculated partial pressures $p_{\mathrm{CO}_{2}}$ for the reference case (Table 3 ) assuming different atmospheric mean temperatures of $300 \mathrm{~K}, 800 \mathrm{~K}$, and $1500 \mathrm{~K}$. Venus and Mars are shown for reference (Venus: $H=15.9 \mathrm{~km}, p_{\mathrm{CO}_{2}}=92 \mathrm{bar}$, $p_{\min }=1 \mathrm{mbar}, T_{\mathrm{atm}}=737 \mathrm{~K}$; Mars: $H=11.1 \mathrm{~km}, p_{\mathrm{CO}_{2}}=6.9 \mathrm{mbar}$, $p_{\min }=1 \mathrm{mbar}, T_{\mathrm{atm}}=210 \mathrm{~K}$ ).

Temperature only evolves as a function of radiogenic heating and cooling from the lithosphere:

$\frac{\partial T}{\partial t}=\frac{Q}{C_{p}}-\frac{\Phi S}{\rho C_{p} V_{m}}$,

where $Q=Q_{0} \exp \left(-t / t_{1 / 2}\right)$ is the radiogenic heating $\left(Q_{0}=\right.$ $2.42 \times 10^{-11} \mathrm{~W} \mathrm{~kg}^{-1}$ and the half life $\left.t_{1 / 2}=2.85 \mathrm{Gyr}\right), C_{p}$ is the

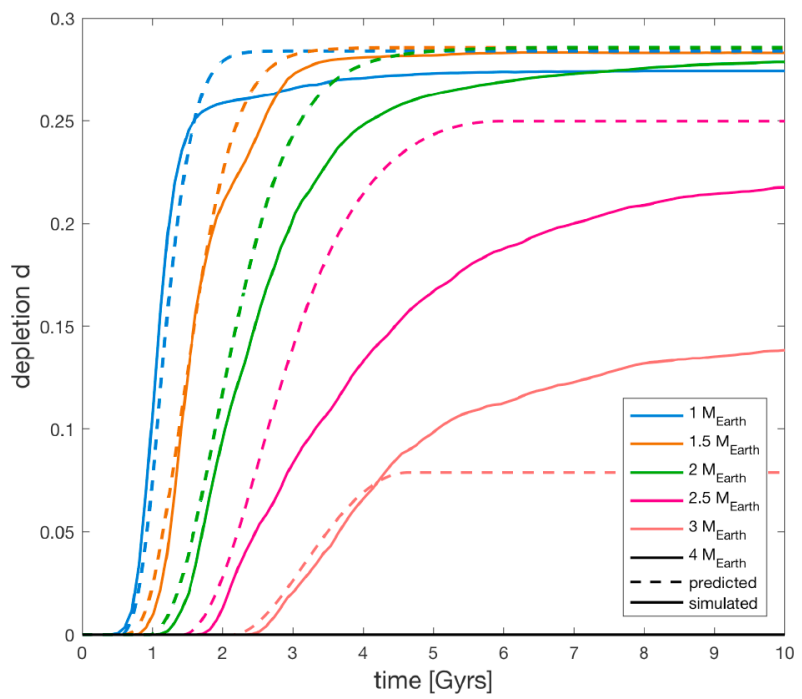

Fig. 18. Time dependence of mantle depletion for selected planet masses (reference case). Solid lines show simulated mantle depletion, whereas dashed lines show predicted values based on our timedependent scaling law.

heat capacity $\left(C_{p}=1200 \mathrm{~J} \mathrm{~kg}^{-1} \mathrm{~K}^{-1}\right), \Phi$ is the (time-dependent) heat flux, $S$ is the surface of the planet, $\rho$ is the average density of the planet and $V_{m}$ is the volume of the mantle.

The heat flux is computed using the standard boundary layer theory:

$\Phi \propto \Phi_{\text {diff }} \operatorname{Ra}^{n}$,

where $n=0.28$ is consistent with previous studies (Fowler 1985; Solomatov 1995; Reese et al. 1998) $\Phi_{\text {diff }}$ is the diffusive heat flux at the surface (in the absence of convection):

$\Phi_{\text {diff }} \propto \frac{R_{\oplus}}{R}$,

where we considered a fixed "equilibrium" surface to core temperature difference for simplicity. To compute the Rayleigh number, we consider the planet mass-dependence of the average thermal expansivity, density, gravity and mantle thickness (assumed half of the planet radius):

$R=R_{\oplus} M^{0.26}$,

$\alpha=\alpha_{\oplus} M^{-1.43}$,

$\rho=\rho_{\oplus} M^{0.22}$,

$g=g_{\oplus} M^{0.48}$

where $M$ is the normalized planet mass $M=M_{p} / M_{\oplus}$. The scaling for the radius was previously derived in Sect. 4.1. Gravity $g$ was obtained using $g=G M_{p} / R^{2}$. The average density was estimated by dividing planet mass by planet volume (thus assuming that the compressibility of mantle and core are similar). The scaling for thermal expansivity $\alpha$ follows Katsura et al. (2010): $\alpha \propto \alpha_{\oplus}\left(\rho_{\oplus} / \rho\right)^{\delta_{T}}$ with $\delta_{T} \approx 6$. The viscosity below the lithosphere is approximated by:

$\eta=\eta_{0} \exp \left(\frac{E}{R_{b}}\left(\frac{1}{T}-\frac{1}{T_{0}}\right)\right)$,

where $\eta_{0}$ is a reference viscosity, $E$ is the activation energy ( $E=300 \mathrm{~kJ} \mathrm{~mol}^{-1}$ ), $R_{b}$ is the universal gas constant and $T_{0}$ is 
a reference temperature $\left(T_{0}=1800 \mathrm{~K}\right)$. The Rayleigh number can then be defined (Travis \& Olson 1994):

$\mathrm{Ra}=\frac{\alpha \rho g(R / 2)^{5} Q}{\kappa \eta} \propto M^{0.57} \exp \left(-\frac{t}{t_{1 / 2}}-\frac{E}{R_{b}}\left(\frac{1}{T}-\frac{1}{T_{0}}\right)\right)$.

Using Eqs. (17), (18) and (24), the heat flux becomes:

$\Phi=\Phi_{0} M^{-0.1} \exp \left(-\frac{0.3 t}{t_{1 / 2}}-\frac{0.3 E}{R_{b}}\left(\frac{1}{T}-\frac{1}{T_{0}}\right)\right)$,

where $\Phi_{0}$ is a constant. We found that a reference heat flux $\Phi_{0}=10 \mathrm{~mW} \mathrm{~m}^{-3}$ best fits the time-dependent formulation. Using Eqs. (15), (16), and (25), we were able to estimate the evolution of the temperature below the lithosphere for all planet masses.

\subsection{Evolution of the depletion}

The depletion $d$ is considered to be the volume sum of depletions in the $\mathrm{CO}_{2}$ producing region (top) and in the rest of the mantle (bot):

$d=\frac{d_{\mathrm{top}} V_{\mathrm{top}}+d_{\mathrm{bot}}\left(V_{m}-V_{\mathrm{top}}\right)}{V_{m}}$,

where $V_{m}$ is the volume of the mantle and $V_{\text {top }}$ is the volume of the $\mathrm{CO}_{2}$ producing region defined by:

$V_{\mathrm{top}}=\frac{4 \pi}{3}\left((R-l)^{3}-R_{c o}^{3}\right)$.

$R_{c o}$ is the cross-over radius above which melt becomes lighter than the solid and $l$ is the lithosphere thickness obtained using the heat flux:

$l=0.78 \frac{(T-300) k}{\Phi}$,

with $k$ being the thermal conductivity $\left(k=3 \mathrm{~W} \mathrm{~m}^{-1} \mathrm{~K}^{-1}\right)$. The factor 0.78 slightly diminishes the lithosphere thickness to account for radiogenic heating in the lithosphere and the potential topography of the base of the lithosphere. It was found necessary to slightly diminish the lithosphere thickness to obtain a consistent temperature evolution and volume of the $\mathrm{CO}_{2}$-producing region.

The depletion in the top region is obtained at each time $t$ in two stages. First, depletion $d_{\text {top }}$ is updated using the melt fraction $\phi$, itself derived from the temperature:

$$
\begin{aligned}
\phi & =\frac{T-T_{\mathrm{s}}}{T_{1}-T_{\mathrm{s}}}, \\
d_{\mathrm{top}}(t) & =\max \left(d_{\mathrm{top}}(t-\Delta t), 0.3 \phi\right),
\end{aligned}
$$

where $T_{\mathrm{s}}$ is the solidus temperature (assumed to be $2100 \mathrm{~K}$ for simplicity) and $T_{1}$ is the liquidus temperature $\left(T_{1}=2300 \mathrm{~K}\right)$. The melt fraction $\phi$ is kept between 0 and 1. The depletion of the previous time step is used as a minimum to prevent depletion from disappearing if the melt fraction decreases.

In each time step, the decrease of depletion is possible through the second stage in which exchange of mass between top and bottom mantle is explicitly estimated. Both top and bottom depletions are updated together using time substeps. An advective depletion flux is considered at the base of the $\mathrm{CO}_{2}$-producing region. The evolutions of depletion take the form:

$$
\begin{aligned}
\frac{\partial d_{\mathrm{bot}}}{\partial t} & =1.2 \frac{V_{\mathrm{top}}}{V_{m}-V_{\mathrm{top}}} \frac{1}{R_{p}-r_{\mathrm{core}}} v\left(d_{\mathrm{top}}-d_{\mathrm{bot}}\right), \\
\frac{\partial d_{\mathrm{top}}}{\partial t} & =-\frac{\partial d_{\mathrm{bot}}}{\partial t} \frac{V_{\mathrm{top}}}{V_{m}-V_{\mathrm{top}}},
\end{aligned}
$$

where $v$ is a velocity consistent with the heat flux as prescribed by classical boundary layer theory (Fowler 1985; Solomatov 1995; Reese et al. 1998):

$v=v_{0}\left(\frac{\Phi}{\Phi_{0}}\right)^{2}$

where $v_{0}$ is fixed to $1 \mathrm{~cm} \mathrm{yr}^{-1}$. Equation (31) shows that the propagation of depleted material from the $\mathrm{CO}_{2}$ producing region to the rest of the mantle does not only depend on the velocity over the thickness of the mantle. The volume ratio of top to bottom layers has to be considered as well. Indeed, if the $\mathrm{CO}_{2}$-producing region is very thin, only a thin layer of depleted material will propagate in the mantle. The evolution of the depletion in the top (Eq. (31)) is equal to minus the evolution in the bottom multiplied by the volume ratio to conserve the mass of depleted material during advection.

Figure 18 shows the resulting evolution of depletion in which it has been considered that the top $100 \mathrm{~km}$ is fully depleted; depletion has therefore been multiplied by the volume of the mantle below $100 \mathrm{~km}$ depth over the total volume of the mantle.

In Fig. 19, we show the time-evolution of depletion and outgassing for various planet masses (of the reference case). The curves of depletion were calculated using our analytical formulation described above. The amount of outgassed $\mathrm{CO}_{2}$ was then calculated using Eq. (12). For ages below 4.5 Gyr, we see that planetary age has a first-order influence on depletion and $p_{\mathrm{CO}_{2}}$. Variations beyond $4.5 \mathrm{Gyr}$ are small. This is important given that observed exoplanets have a wide range of ages.

\subsection{Comparison between the time-dependent depletion model and the scaling law for depletion for a fixed 4.5-Gyr evolution}

Since the evolution of depletion relies on the numerical integration of partial differential equations, Eq. (9) does not provide an explicit time-dependent prediction for depletion. Yet the necessity for most terms in Eq. (9) can be better understood considering the driving processes in our time-dependent formulation.

The first term of Eq. (9) shows that depletion is strongly related to the relative volumes of the $\mathrm{CO}_{2}$-producing region and the overall mantle. In our time-dependent formulation, these relative volumes also play a central role. We observed that the time-dependence of the existence of this top region is necessary to reproduce the onset times of depletion (see Fig. 18). The existence of the top region strongly depends on both lithosphere thickness and internal temperature which are both strongly linked to the surface heat flux. The fact that the heat flux derived from boundary layer theory enables us to reproduce the time-dependence of our simulations is remarkable and gives us confidence that boundary layer theory can be used in the investigation of exoplanet evolution. Yet, several terms of our scaling 

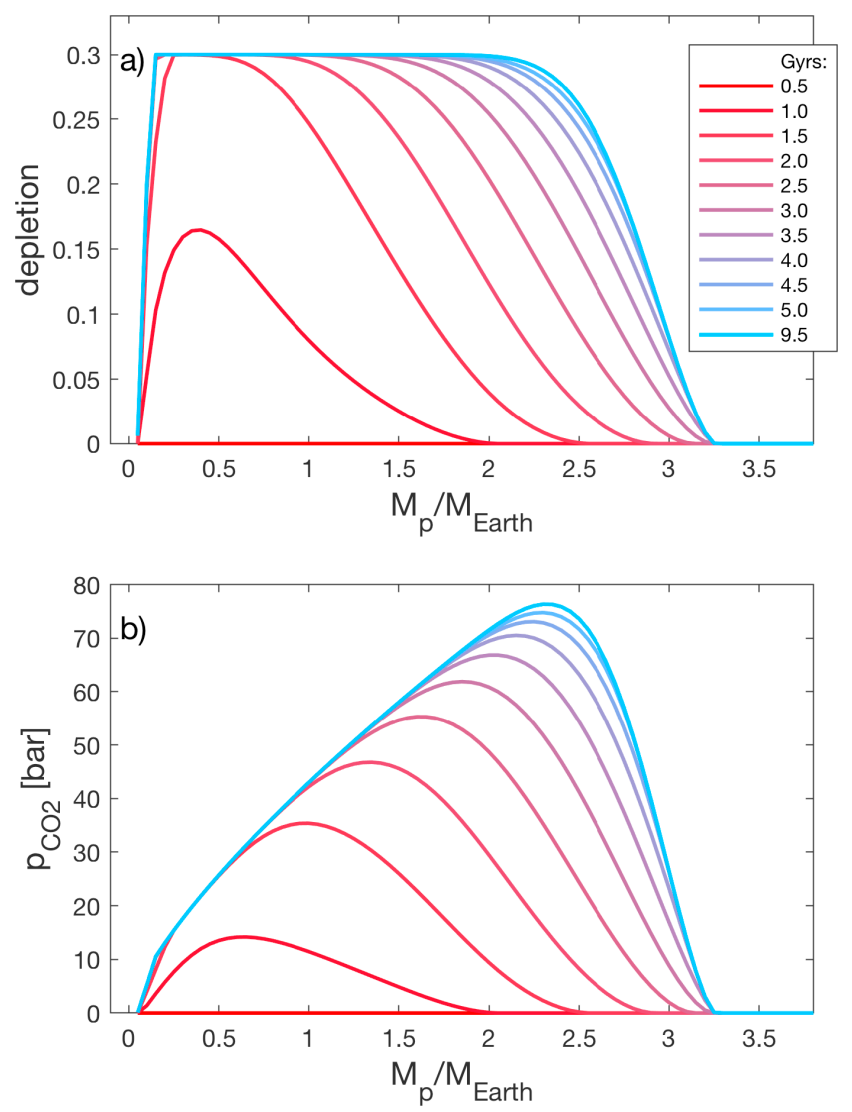

Fig. 19. Time dependence of $a$ ) mantle depletion and $b$ ) amount of outgassed $\mathrm{CO}_{2}$ as a function of planet mass (reference case). Depletion has been calculated with our analytical formulations in Sect. 6, and Eq. (12) was subsequently used to calculate the amount of outgassed $\mathrm{CO}_{2}$.

law for depletion (Eq. (9)) are highly nonlinear and cannot easily be derived from our time-dependent formulation, which requires the use of convection simulations.

The second term of Eq. (9) can also easily be understood using our time-dependent formulation. The occurrence of melting and $\mathrm{CO}_{2}$ degassing depends on whether or not the solidus temperature is reached. Equation (15) demonstrates the importance of the initial temperature to reach the solidus temperature $T_{\mathrm{s}}$.

The third term of Eq. (9) shows that internal heating also governs the occurrence of melting. Again this can easily be understood from Eqs. (15), (16), and (29).

The importance of the viscosity (fourth term in Eq. (9)) in mantle depletion arises from several processes in our timedependent formulation. The heat flux is related to the viscosity as demonstrated by boundary layer theory (Eqs. (17) and (24)). The heat flux plays a central role in both temperature evolution (Eq. (16)) and lithosphere thickness (Eq. (28)). The negativity of the factor $\gamma$ in Eq. (9) shows that an increase in viscosity decreases the depletion. This shows the central role of existence of the $\mathrm{CO}_{2}$ producing region as a large viscosity will result in a low heat flux and a large temperature. Melting is therefore more important (Eqs. (15), (16), and (29)) but as the lithosphere is too thick there is no volcanism and outgassing of $\mathrm{CO}_{2}$.

$\mathrm{The} \mathrm{Mg} / \mathrm{Si}$ ratio does not enter our time-dependent formulation. We therefore cannot reproduce the fifth term in Eq. (9). The impact of the $\mathrm{Mg} / \mathrm{Si}$ ratio could probably be understood by investigating its effects on the Rayleigh number through density variations.
The sixth and seventh terms of our scaling law for depletion are highly nonlinear and cannot be understood clearly from our simple time-dependent formulation. These terms show that some combination of internal heating, iron content, and core size have a second-order effect on depletion. The impact of iron content can be understood from our time-dependent formulation as the solidus temperature is strongly Fe-dependent as shown in Sect. 3.2.

In conclusion, the time-dependence of $\mathrm{CO}_{2}$-outgassing in stagnant-lid planets can be understood using boundary layer theory for any planetary mass. One limitation of our model is that crust production is neglected which makes it impossible to observe the recycling of basaltic material in the mantle. This could easily be taken into account in a parameterized model by estimating the amount of basalt produced and comparing its volume to the volume of the lithosphere. If the volume of basalt exceeds the volume of the lithosphere, then the depletion of the mantle should be decreased as enriched material should be dripping back in the mantle from the base of the lithosphere. This would simply result in adding a source term to the depletion in the $\mathrm{CO}_{2}$ producing region. However, this limitation has no impact on the large planets which nonetheless seem to never be able to produce basaltic material.

\section{Discussion}

Interior dynamics and outgassing are linked to interior properties. The anticipated variability of super-Earth interior structures and compositions can be partly informed by commonly observed astrophysical data from exoplanets. These data include planetary mass and radius, and bulk abundances of rock-forming elements (i.e., $\mathrm{Fe}, \mathrm{Mg}, \mathrm{Si}$ ). In addition, we expect a wide variability on key thermal parameters that are very difficult to constrain using observations. On this basis, we compiled a set of superEarths that incorporates the anticipated variability of structural, compositional, thermal parameters, and age of the majority of super-Earths. This set excludes super-Mercuries, that are distinct from super-Earths by larger core-mantle ratios. It is yet unclear how frequent super-Mercuries are. Thus, our study goes beyond a simple parameter study where only one parameter is altered at a time. Our test cases are chosen such that they incorporate the current knowledge on rocky exoplanet interiors.

This study is a significant step towards interpreting astrophysical observations of exoplanet atmospheres by geophysical interior models. Any interpretation of astrophysical observations of super-Earth atmospheres must be done in light of relevant formation and evolution processes of atmospheres. Here, we have focused on long-term outgassing processes that shape the atmosphere of terrestrial-type stagnant-lid exoplanets but have neglected other processes such as (1) the early outgassing from a magma ocean, (2) atmospheric erosion due to stellar irradiation, (3) weathering, and (4) any primordial hydrogen atmosphere. Considering these complexities, our results represent first-order estimates. We briefly discuss these aspects in the following.

\subsection{Outgassing from magma oceans}

Early outgassing from a magma ocean (e.g., Elkins-Tanton 2008; Lebrun et al. 2013) can be in principle incorporated by choosing initial nonzero values of $p_{\mathrm{CO}_{2}}$. It is possible that such initial amounts of primary atmospheres exceed the variations due to the long-term volcanism by up to several hundred bars (Lebrun et al. 2013). 


\subsection{Atmospheric escapes}

Although atmospheric escape can efficiently erode hydrogen atmospheres, the erosion of $\mathrm{CO}_{2}$ is much more inefficient, which is also because the high mean-molecular weight of $\mathrm{CO}_{2}$ (Lopez 2017). In addition, the Super-Earths of interest are temperate planets for which stellar irradiation is limited. If erosion of outgassed atmospheres is significant, than it is the early outgassed atmosphere from a magma ocean that is mainly effected, since the stellar high-energy irradiation is strongest during the early evolution of a star.

\subsection{Weathering}

Outgassed $\mathrm{CO}_{2}$ can also be removed from the atmosphere via carbonate weathering. However, weathering requires sufficient supply of fresh, weatherable rock, which is limited for stagnantlid regimes (Foley \& Driscoll 2016). Foley \& Smye (2018) argue that weathering can significantly limit atmospheric $\mathrm{CO}_{2}$ accounting for supply-limited weathering. However, given the possible variability in Super-Earth compositions, the variability in carbonation efficiency of different erupted rocks requires further understanding.

Other sinks of $\mathrm{CO}_{2}$ are water oceans; however, the carbon ocean reservoir is small compared to the mantle reservoir (Sleep \& Zahnle 2001). The solubility of $\mathrm{CO}_{2}$ in water is temperature dependent and increases as temperatures drop (e.g., Kitzmann et al. 2015; Pierrehumbert 2010). In principle, our predicted amounts of outgassed $\mathrm{CO}_{2}$ can be used as input in climate models to investigate whether $\mathrm{CO}_{2}$ would be present as gas in the atmosphere, as ice on the surface, or partially dissolved in a possible water ocean (e.g., Menou 2015; Abbot et al. 2012; Tosi et al. 2017). For Earth-sized stagnant-lid planets, Foley \& Smye (2018) suggest that $\mathrm{CO}_{2}$ budgets low enough to prevent runaway greenhouse and high enough to prevent global glaciation range from $10^{-2}-1$ times the Earth's budget.

\subsection{Primordial hydrogen atmosphere}

Any primordial hydrogen-dominated atmospheres could in principle make the identification of outgassed atmospheres difficult. Fortunately, even if spectroscopic investigations of a SuperEarth's atmosphere (e.g., Bourrier et al. 2017; Benneke et al. 2017; Knutson et al. 2014) are not available, considerations of atmospheric escape (Dorn \& Heng 2018) can provide necessary constraints in addition to mass and radius to distinguish between hydrogen-dominated and enriched (e.g., outgassed) atmospheres. Thereby, the thickness or mass fraction of a gas envelope that is likely outgassed from the interior can be quantified (Dorn \& Heng 2018) and misinterpretations due to the presence of a hydrogen-dominated envelope can be reduced.

\subsection{Observational constraints on outgassed atmospheres}

Characterizing interiors and atmospheres of exoplanets is a highly degenerate problem. However, it is possible to quantify probabilities of atmospheric properties (i.e., mass and radius fraction of an atmosphere and its enrichment in heavier molecules) as demonstrated by Dorn \& Heng (2018). They determine that enriched (and possibly outgassed) atmospheres preferably occur on planets of small masses and high equilibrium temperatures. Their use of a generalized Bayesian inference analysis allowed them also to quantify the atmosphere thicknesses for a set of about 20 exoplanets. Interpreting such a distribution of possibly outgassed atmospheres requires geophysical interior models. Our study provides a significant part of the necessary tools to make an informed interpretation.

Improved estimates on the distributions of possibly outgassed atmospheres are expected to be made possible by the data of upcoming missions (e.g., TESS, CHEOPS, JWST). These missions will not only significantly increase the number of exoplanet detections (e.g., TESS), but also provide better precision on the data that we use to characterize their interiors (e.g., CHEOPS) and make it possible to probe, in detail, the atmospheres of some tens of Super-Earth (JWST).

If observations were to confirm our predicted trend of $\mathrm{CO}_{2}$ atmospheres with planet mass, this would suggest that the majority of Super-Earths are in a stagnant-lid regime. Deviating behaviors may be explained by dynamic regimes other than stagnant-lid; for example, plate tectonics (Valencia et al. 2007a; Kite et al. 2009; Korenaga 2010; Van Heck \& Tackley 2011; Noack et al. 2014; O’Neill \& Lenardic 2007; Lenardic \& Crowley 2012; Foley et al. 2012) or atmospheres being dominated by the early outgassing during the cooling of a magma ocean (Hamano et al. 2013).

Commonly observed exoplanets orbit at close distances to their stars which involves much higher surface temperatures than our assumed $280 \mathrm{~K}$ fixed value. In fact, surface temperatures of observed exoplanets may allow for surface rocks to be molten. Analyzing outgassing under such temperature conditions would require the modeling of a magma ocean, crustal production, and melt migration processes, which is beyond the scope of this paper. Here, we focused on temperate exoplanets, for which upcoming missions (e.g., TESS, CHEOPS, JWST) will provide data for interior characterization, for example from planets around M-dwarf stars (e.g., Trappist-1 system).

\subsection{Impact on habitability}

The classical definition of the habitable zone assumes the availability of greenhouse gases such as $\mathrm{CO}_{2}$ (e.g., Kasting et al. 1993; Kopparapu et al. 2014). The outer boundary of the habitable zone mostly depends on the amount of $\mathrm{CO}_{2}$, while the inner boundary of the habitable zone is characterized by both the amounts of $\mathrm{CO}_{2}$ and $\mathrm{H}_{2} \mathrm{O}$ (Tosi et al. 2017). Since volcanism maintained over geological time-scales is possible for stagnantlid planets, it is suggested that these planets can be habitable (e.g., Noack et al. 2017; Tosi et al. 2017; Foley \& Smye 2018).

Our results show that volcanism is limited for stagnant-lid planets of masses larger than 5-7 $M_{\oplus}$ or older than 5 Gyr. This suggests that volcanic activity suitable for habitability is restricted to small planets $\left(<5-7 M_{\oplus}\right)$ as well as planets younger than $\sim 5$ Gyr. This is in agreement with previous studies (Noack et al. 2017; Foley \& Smye 2018).

Habitability depends also on the presence of other greenhouse gases that affect the surface temperature. Changes in surface temperatures feed back to the deformability of the lithosphere (e.g., Bercovici \& Ricard 2014) and thus to outgassing. Possible greenhouse gases other than $\mathrm{CO}_{2}$ that can drive this thermal feedback include for example $\mathrm{H}_{2} \mathrm{O}$. The efficiency of these feedback mechanisms depends on atmospheric amounts of the gases and their recycling dynamics between mantle and atmosphere. Here, we focused on the outgassing of $\mathrm{CO}_{2}$ only. However, the limitations in volcanic activity discussed in our study similarly affect the outgassing of gases other than $\mathrm{CO}_{2}$. For example, the solubility of $\mathrm{H}_{2} \mathrm{O}$ in melt is much higher than for $\mathrm{CO}_{2}$. Therefore, partial pressures of outgassed $\mathrm{H}_{2} \mathrm{O}$ can be one 
order of magnitude smaller compared to $\mathrm{CO}_{2}$, while evolution trends of outgassing are similar (Tosi et al. 2017).

\subsection{Scaling law}

We developed scaling laws to summarize the efficiency of mantle outgassing depending on several super-Earth characteristics. The functional form of our derived scaling laws is based on physics and involves free fitting parameters. Our scaling law is able to describe the two trends of outgassing as a function of planet mass: at low mass, the outgassing increases with mass, whereas it decreases at higher masses. We showed that thermal, structural, and compositional parameters can alter the transition between these two trends. We expect that other parameters that we did not consider could similarly affect this transition, however, they would not influence the existence of both trends. For example, rheological variability due to different grain sizes, hydration, compositions, and melt fraction are neglected in the present study.

\subsection{Thermal convection model}

As commonly done, the investigated stagnant-lid regime is based on pure thermal convection and excludes the dynamical effects of crust production that involves production and eruption of melt (e.g., Kite et al. 2009; Noack et al. 2012). Crust production is rarely modeled in global mantle convection simulations since it is computationally more expensive (see Xie \& Tackley 2004; Keller \& Tackley 2009; Nakagawa et al. 2010, for implementations), although Moore \& Webb (2013); Lourenço et al. (2016) reported that melting and (basaltic) crust production can have a first-order impact on the convection regime of Earth-like planets. For example, strong enough eruptive magmatism can turn a stagnant-lid regime into an episodic regime (Lourenço et al. 2016).

Yet, melting and crust production will not always affect the convection regime. Here, we showed that partial melting is limited for very large exoplanets that are in a stagnant-lid regime. Although magmatism might be important for Earth-sized planets, it could be negligible for smaller- (Mars) or higher-mass (super-Earths) planets. Small planets cool much faster which makes melting only important in early stages, as has been shown for Mars (Taylor \& McLennan 2009). For high planet masses there are two effects that lead to reduced depletion. The first effect is the decrease in density cross-over depth with mass (see details in Sect. 4.5). The second effect is the increase of melting temperature with pressure. Thus, for high-mass planets, the melting temperature beneath the lithosphere is generally higher than the adiabatic temperature which prevents melting. In such cases, magmatism might be restricted to planets with very thin lithosphere thicknesses that can develop in regimes such as plate tectonics. In the future, further investigations will be necessary to better understand the effect of different tectonic regimes on outgassing.

\section{Conclusions}

The atmospheres of the terrestrial Solar System planets are shaped by volcanic outgassing that occurs on geological timescales, which we also expect to be relevant for superEarth atmospheres. Furthermore, the atmospheres are the only parts of exoplanets that can be directly probed and upcoming missions (e.g., JWST, E-ELT) will provide detailed insight into exoplanet atmospheres. The interpretation of super-Earth atmospheres crucially relies on our understanding of volcanic outgassing. Here, we have thoroughly studied the diversity of outgassing on stagnant-lid super-Earths given the anticipated diversity of their interiors. Thus, our study informs upcoming findings of observed super-Earth atmospheres.

Specifically, we investigated the amount of outgassed $\mathrm{CO}_{2}$ given the anticipated diversity of super-Earths interiors. We built on the work of Noack et al. (2017) and assumed a stagnant-lid convection regime. We accounted for a broad range of possible interiors of rocky exoplanets $\left(1-8 M_{\oplus}\right)$ that are in agreement with commonly observed astrophysical constraints of mass, radius, and stellar abundances. Stellar abundances of refractory elements are candidates for placing constraints on the relative abundance of rock-forming elements (i.e., $\mathrm{Mg}, \mathrm{Si}, \mathrm{Fe}$ ) in the planet bulk. We also accounted for possible variations in interior parameters that are very difficult if not impossible to constrain from astrophysical data. These mostly include initial and thermal parameters, for example, the amount of radiogenic heat sources, the initial mantle temperature, or additional heat flux from the core; other investigated parameters are compositionrelated effects such as viscosity, influence of water, and density cross-over pressure. The surface temperatures were assumed to be Earth-like.

Our results are comparable to those of Noack et al. (2017), where a simple silicate mantle and pure iron core composition was used to show that outgassing ceases at high planetary masses.

Based on our large number of 2340 super-Earth models, we conclude the following:

- Planetary mass $M_{\mathrm{p}}$ mainly influences the amount of outgassing on stagnant-lid planets. At small masses $\left(<2 M_{\oplus}\right.$, for the reference case), maximum mantle depletion is reached and outgassing positively correlates with planet mass, since it is controlled by the absolute mantle volume. At large masses ( $>2 M_{\oplus}$, for the reference case), depletion and thus outgassing decreases with planet mass, which is due to the increasing pressure gradient that leads to an increasing melting temperature beneath the lithosphere and limits melting to shallower depths. For stagnantlid planets above $\sim 7 M_{\oplus}$, the large pressure gradient and the high melting temperatures beneath the lithosphere generally prohibit partial melting at depth. Thus, for stagnant-lid planets, we expect that (1) there is a mass range of planets for which outgassing is most effective and (2) there is an upper mass limit above which outgassing rarely occurs. This predicted trend of $\mathrm{CO}_{2}$ atmospheres with planet mass can be observationally tested for exoplanets. Deviating behaviors may be explained by dynamic regimes other than stagnant-lid, for example, plate tectonics, or atmospheres being dominated by the early outgassing during the cooling of a magma ocean. The distribution of enriched atmospheres can be observationally tested with upcoming missions that aim at characterizing exoplanet atmospheres (e.g., JWST, E-ELT).

- Thermal parameters can significantly shift the mass range where maximum outgassing can occur and thus shift the transition between positive and negative correlation between $M_{\mathrm{p}}$ and $p_{\mathrm{CO}_{2}}$. We find that by varying the initial mantle temperature from $1600 \mathrm{~K}$ to $2000 \mathrm{~K}$, this shift is on the order of $1 M_{\oplus}$, whereas the variation from 0.5 to 1.5 times the amount of Earthlike radiogenic heat results in a shift of the order of $3 M_{\oplus}$. The tested ranges of thermal parameters broadly covers the expected variability among stagnant-lid exoplanets.

- The anticipated range of exoplanet ages is wide and on the scale of Gigayears. Although, most of our results summarize the outgassing after 4.5 Gyr of evolution, we discuss the evolution 
of volcanism up to $10 \mathrm{Gyr}$ (in Sect. 6). For ages below $4.5 \mathrm{Gyr}$, planetary age can have first-order influence on depletion and the amount of outgassed $\mathrm{CO}_{2}$. However, outgassing beyond $4.5 \mathrm{Gyr}$ only adds small or negligible amounts of $\mathrm{CO}_{2}$ to an atmosphere. Our investigation shows that planets of masses above $3 M_{\oplus}$ (reference case) do not have significant outgassing, even over an extended evolution of $10 \mathrm{Gyr}$.

- Mantle composition seems to be of secondary influence for outgassing. Mantle composition influences melting temperature and mantle density. A more iron-rich mantle material has a lower melting temperature which increases melting and thus leads to higher outgassing ( $<2 M_{\oplus}$, for the reference case). At the same time, an iron-rich mantle composition implies high mantle densities which increase the pressure gradient in the lithosphere. Thus, at large masses ( $>2 M_{\oplus}$, for the reference case), when melting is limited by the pressure gradient as discussed earlier, a more iron-rich mantle tends to outgas less. Composition also influences the viscosity of the mantle, the melting temperature and the density cross-over pressure. While all of these factors tend to influence the amount of outgassing in the intermediate-mass range $\left(2-4 M_{\oplus}\right)$, no significant change in outgassing is observed for low-mass planets, where depletion is efficient for all tested cases as well as for more massive planets, where little or no outgassing occurs.

- The effect of core size is of secondary influence for outgassing. At small masses, where outgassing is controlled by mantle volume, a smaller core size increases the amount of outgassing. At larger masses, we find the opposite trend. Due to the bulk abundance constraints, a smaller core implies an iron-rich and thus dense mantle material, which results in a higher pressure gradient in the lithosphere. Therefore, melting is limited to shallower regions and outgassing is reduced.

- We estimate the respective gas layer thicknesses of the calculated outgassed $\mathrm{CO}_{2}$ and compare them with independent estimates of Venus and Mars and find good agreement.

Finally, we provide scaling laws that summarize the influence of first- and second-order interior parameters on mantle depletion and outgassing on stagnant-lid planets. Thereby, our study represents a significant step towards providing means of interpretation for comparative studies of exoplanet atmospheres.

Acknowledgements. We thank the anonymous reviewer. C.D. received funding from the Swiss National Foundation under grant 200020_160120, PZ00P2_174028, and from the MERAC grant by the Swiss Society of Astrophysics and Astronomy. L.N. received funding from the Interuniversity Attraction Poles Programme initiated by the Belgian Science Policy Office through the Planet Topers alliance. A.B.R. received funding from the European Research Council under the European Union's Seventh Framework Programme (FP/20072013)/ERC Grant Agreement number 320639 project iGEO. This study was in part carried out within the frame of the National Centre for Competence in Research PlanetS

\section{References}

Abbot, D. S., Cowan, N. B., \& Ciesla, F. J. 2012, ApJ, 756, 178

Bajgain, S., Ghosh, D. B., \& Karki, B. B. 2015, Nat. Commun., 6

Benneke, B., Werner, M., Petigura, E., et al. 2017, ApJ, 834, 187

Bercovici, D., \& Ricard, Y. 2014, Nature, 508, 513

Bouchet, J., Mazevet, S., Morard, G., Guyot, F., \& Musella, R. 2013, Phys. Rev. B, 87, 094102

Bourrier, V., Ehrenreich, D., King, G., et al. 2017, A\&A, 597, A26

Burov, E. 2007, Plate Rheology and Mechanics-6.03 (Amsterdam: Elsevier BV)

Byerlee, J. D. 1978, in Rock Friction and Earthquake Prediction (Basel: Birkhäuser Verlag), 116, 615

Connolly, J. A. D. 2009, Geochem. Geophys. Geosyst., 10, 10014

Dorn, C., \& Heng, K. 2018, ApJ, 853, 64

Dorn, C., Khan, A., Heng, K., et al. 2015, A\&A, 577, A83

Dorn, C., Venturini, J., Khan, A., et al. 2017, A\&A, 597, A37
Dressing, C. D., \& Charbonneau, D. 2015, ApJ, 807, 45

Elkins-Tanton, L. T. 2008, Earth Planet. Sci. Lett., 271, 181

Fischer, R., \& Gerya, T. 2016, J. Geodyn., 100, 198

Foley, B. J., \& Driscoll, P. E. 2016, Geochem. Geophys. Geosyst., 17, 1885

Foley, B. J., \& Smye, A. J. 2018, Astrobiology, submitted

Foley, B. J., Bercovici, D., \& Landuyt, W. 2012, Earth Planet. Sci. Lett., 331, 281

Fowler, A. C. 1985, Stud. Appl. Math., 72, 189

Frank, E. A., Meyer, B. S., \& Mojzsis, S. J. 2014, Icarus, 243, 274

Fulton, B. J., Petigura, E. A., Howard, A. W., et al. 2017, AJ, 154, 109

Gaillard, F., \& Scaillet, B. 2014, Earth Planet. Sci. Lett., 403, 307

Gerya, T. 2014, Gondwana Res., 25, 442

Gillmann, C. \& Tackley, P. 2014, J. Geophys. Res. Planet., 119, 1189

Hamano, K., Abe, Y., \& Genda, H. 2013, Nature, 497, 607

Hernlund, J. W., \& Tackley, P. J. 2008, Phys. Earth Planet. Inter., 171, 48

Herzberg, C., Condie, K., \& Korenaga, J. 2010, Earth Planet. Sci. Lett., 292, 79

Hinkel, N. R., Timmes, F., Young, P. A., Pagano, M. D., \& Turnbull, M. C. 2014 AJ, 148, 54

Hirschmann, M. M. 2000, Geochem. Geophys. Geosyst., 1, 1042

Hirth, G., \& Kohlstedt, D. 2003, Inside the Subduction Factory (Washington: American Geophysical Union), 138, 83

Hüttig, C., \& Breuer, D. 2011, Phys. Earth Planet. Inter., 186, 111

Jaupart, C., Labrosse, S., \& Mareschal, J. 2007, Treatise Geophys., 7, 223

Karato, S.-i., \& Wu, P. 1993, Science, 260, 771

Kasting, J. F., Whitmire, D. P., \& Reynolds, R. T. 1993, Icarus, 101, 108

Katsura, T., Yoneda, A., Yamazaki, D., Yoshino, T., \& Ito, E. 2010, Phys. Earth Planet. Inter., 183, 212

Katz, R. F., Spiegelman, M., \& Langmuir, C. H. 2003, Geochem. Geophys. Geosyst., 4, 1073

Keller, T., \& Tackley, P. J. 2009, Icarus, 202, 429

Kiefer, W., Filiberto, J., Sandu, C., \& Li, Q. 2015, Geochim. Cosmochim. Acta, 162,247

King, S. D., Lee, C., Van Keken, P. E., et al. 2010, Geophys. J. Int., 180, 73

Kite, E. S., Manga, M., \& Gaidos, E. 2009, ApJ, 700, 1732

Kitzmann, D., Alibert, Y., Godolt, M., et al. 2015, MNRAS, 452, 3752

Knutson, H. A., Dragomir, D., Kreidberg, L., et al. 2014, ApJ, 794, 155

Kohlstedt, D., Evans, B., \& Mackwell, S. 1995, J. Geophys. Res. Solid Earth, 100, 17587

Kopparapu, R. K., Ramirez, R. M., SchottelKotte, J., et al. 2014, ApJ, 787, L29

Korenaga, J. 2010, ApJ, 725, L43

Lebrun, T., Massol, H., Chassefière, E., et al. 2013, J Geophys. Res. Planet., 118 1155

Leconte, J., Forget, F., \& Lammer, H. 2015, Exp. Astron., 40, 449

Lenardic, A., \& Crowley, J. W. 2012, ApJ, 755, 132

Lodders, K. 2003, ApJ, 591, 1220

Lopez, E. D. 2017 MNRAS, 472, 245

Lourenço, D., Rozel, A., \& Tackley, P. 2016, Earth Planet. Sci. Lett., 438, 18

Mackwell, S., Zimmerman, M., \& Kohlstedt, D. 1998, J. Geophys. Res. Solid Earth, 103, 975

Mallard, C., Coltice, N., Seton, M., Mueller, D., \& Tackley, P. 2016, Nature, 535, 140

Marcus, R. A., Sasselov, D., Hernquist, L., \& Stewart, S. T. 2010, ApJ, 712, L73

Marsh, B. 2010, Crust and Lithosphere Dynamics: Treatise on Geophysics, ed. A. B. Watts (Amsterdam: Elsevier BV), 6, 275

McDonough, W. F., \& Sun, S.-S. 1995, Chem. Geol., 120, 223

Menou, K. 2015, Earth Planet. Sci. Lett., 429, 20

Moore, W. B., \& Webb, A. A. G. 2013, Nature, 501, 501

Moresi, L., \& Solomatov, V. 1998, Geophys. J., 133, 669

Nakagawa, T., Tackley, P. J., Deschamps, F., \& Connolly, J. A. 2010, Earth Planet. Sci. Lett., 296, 403

Noack, L., \& Breuer, D. 2014, Planet. Space Sci., 98, 41

Noack, L., Breuer, D., \& Spohn, T. 2012, Icarus, 217, 484

Noack, L., Godolt, M., von Paris, P., et al. 2014, Planet. Space Sci., 98, 14

Noack, L., Rivoldini, A., \& Van Hoolst, T. 2017, Phys. Earth Planet. Inter., 269, 40

O’Neill, C., \& Lenardic, A. 2007, Geophys. Res. Lett., 34

Papuc, A. M., \& Davies, G. F. 2008, Icarus, 195, 447

Pierrehumbert, R. T. 2010, Principles of Planetary Climate (Cambridge: Cambridge University Press)

Reese, C. C., Solomatov, V. S., \& Moresi, L.-N. 1998, J. Geophys. Res., 103 13643

Reese, C., Solomatov, V., Baumgardner, J., \& Yang, W.-S. 1999, Phys. Earth Planet. Inter., 116, 1

Rozel, A., Golabek, G., Jain, C., Tackley, P., \& Gerya, T. 2017, Nature, 545, 332 Sakamaki, T., Suzuki, A., \& Ohtani, E. 2006, Nature, 439, 192

Schubert, G., Turcotte, D. L., \& Olson, P. 2001, Mantle Convection in the Earth and Planets (Cambridge: Cambridge University Press), 956

Schwartz, S., Allemand, P., \& Guillot, S. 2001, Tectonophysics, 342, 193

Silva Aguirre, V., Davies, G., Basu, S., et al. 2015, MNRAS, 452, 2127 
Sizova, E., Gerya, T., Stüwe, K., \& Brown, M. 2015, Precambr. Res., 271, 198 Sleep, N. H., \& Zahnle, K. 2001, J. Geophys. Res. Planet., 106, 1373

Solomatov, V. S. 1995, Phys. Fluids, 7, 266

Stein, C., Schmalzl, J., \& Hansen, U. 2004, Phys. Earth. Plan. Inter., 142, 225

Stixrude, L. 2014, Phil. Trans. R. Soc. London, Ser. A, 372, 20130076

Strom, R., Schaber, G., \& Dawson, D. 1994, J. Geophys. Res., 99, 10899

Tackley, P. J., Ammann, M., Brodholt, J. P., Dobson, D. P., \& Valencia, D. 2013 , Icarus, 225,50

Taylor, S. R., \& McLennan, S. 2009, Planetary Crusts: Their Composition, Origin and Evolution (Cambridge: Cambridge University Press)
Tosi, N., Godolt, M., Stracke, B., et al. 2017, A\&A, 605, A71

Travis, B., \& Olson, P. 1994, Geophys. J. Int., 118, 1

Valencia, D., \& O'Connell, R. J. 2009, Earth Planet. Sci. Lett., 286, 492

Valencia, D., O'Connell, R. J., \& Sasselov, D. D. 2007a, ApJ, 670, L45

Valencia, D., Sasselov, D. D., \& O'Connell, R. J. 2007b, ApJ, 665, 1413

Van Heck, H., \& Tackley, P. 2011, Earth Planet. Sci. Lett., 310, 252

Vilella, K., \& Kaminski, E. 2017, Phys. Earth Planet. Inter., 266, 18

Xie, S., \& Tackley, P. J. 2004, J. Geophys. Res., 109, B11204

Zhao, Y.-H., Zimmerman, M. E., \& Kohlstedt, D. L. 2009, Earth Planet. Sci. Lett., 287, 229 